\title{
Dic unsichtbare „Cupola“: Kausalitätskrise und kollektive Zurechnung*
}

\section{Dic Krise der juristischen Kausalität}

Nicht Brunelleschis architektonisches Meisterwerk, die Kuppel des Florenzer Doms, erscheint vor meinen Augen, wenn ich von der "Cupola“ spreche. Viclmehr sehe ich eine häßliche, dunkle Architcktur, dic brutale Cupola des organisierten Verbrechens, die geheime hierarchische Spitze der Mafia, welche das soziale Leben Italiens überschattet. Gibt es die Cupola oder gibt es sie nicht? Diese Frage hat die italienische Justiz in einem bitteren Streit ent $z$ weit. Während dic crstinstanzlichen Gerichte zahlreiche Mafiosi allein deswegen zu langen Gefängnisstrafen verurteilt haben, weil sic der Cupola, die über „tutti i grandi delitti" entscheidet, als Mitglieder angehörten, hat das Gericht der höheren Instanz über Jahre hinweg die schicre Existenz dieser Cupola verneint und die „Paten“ des organisierten Verbrechens wieder auf freien Fuß gesetzt. Ist die Cupola cin Phantom, cin Phantasieprodukt paranoider Richter? Oder ist sie eine harte soziale Realität, die Juristen zur Kenntnis zu nehmen haben, wenn sie organisiertes Verbrechen und Makrokriminalität überhaupt verstehen wollen?

Natürlich ist sie für das Recht weder das eine noch das andere, weder blasse Fiktion noch harte Realität. Die Cupola ist cine Konstruktion der Rechtsarchitektur, die ihre Existenz dem cinen Zwecke verdankt, Individuen schon dann strafrechtlich zur Verantwortung ziehen zu können, wenn nur ihre Mitgliedschaft in dieser Quasi-Organisation juristisch bewiesen werden kann. Wer Mitglied der Cupola ist, wird zum Mittäter des mafiosen Delikts, ohne daß eine konkrete Tatbeteiligung

I Iür wertvolle Mitarbeit und Kritik danke ich Martin Hohlweck, EUI Floren\%. 
nachgewiesen werden muk. In der Tat, die Cupola ist eine gewagte Konstruktion. Wie aber könnte man sonst der "MakroKriminalitat" beikommen? Dic Rechtskonstruktion der Cupola hilft in der Krise der Kausalzurechnung. Sie befreit von den fast unüberwindlichen Schwierigkeiten des individuellen Kausalnachweises und ersetzt kausale durch kollektive Zurechnung. Unter dem Dach der Cupola verwandelt sich Individualhaftung in Kollektivhaftung ${ }^{1}$.

Heute zeichnen sich die Konturen einer ähnlichen Cupola auch im Umwelthaftungsrecht ab. Das Bemühen der dortigen Rechtskonstrukteure, dic Architektur der Umwelthaftung auf einer stabilen Struktur kausaler Verstrebungen zwischen individuellen Handlungen und ökologischen Schäden zu errichten, wurde durch die Komplexität und Intransparenz der kausalen Wirkungszusammenhänge in den drei ökologischen Medien Luft, Wasser, Boden - zutiefst frustriert. Zusehends schwindet das Vertrauen der Umweltjuristen in die Belastbarkeit der Kausalarchitektur. Sie probieren stattdessen allerhand Hilfskonstruktionen, die zwar in der Theorie noch auf der Kausalstruktur beruhen, in der Praxis sich aber nicht mehr auf individuelle Kausalzurechnung verlassen. Anzeichen dafür sind etwa die immer stärkere Betonung eines spezifisch juristischen Kausalitätsbegriffs, der prima-facic-Beweis, „enhanced res ipsa loquitur", die Beweislastumkehr für Kausalität, der probabilistische Kausalitätsnachweis, dic Ausdehnung gesamtschuldnerischer Haftung bei Multikausalität, "market share liability“ und Superfund-Haftung. All dies sind neue Formen von "Risikohaftung "2, welche die Kausalverbindungen zwischen Handlung und Schaden schwächen oder ganz kappen. Die neue Ri-

1 Im italienischen Recht gibt es neben der Cupola- Konstruktion noch eine andere Form der Kollektivhafung: ,associazione per delinquere astociazione di tipo mafioso" (art. 416 codice penale). Hier ist die Mitghedschaft in einer solchen Vereingung strabar, whithend bei der Cupola-Haftung ein Delikt vorausyesetht ist, bei den dan der konkrete Ththeitrag des Mituaters durch die Mitgliedschate in der Cupola ersent wird.

2 Robinson (1985). Die Einzdheiten der verschiedsuen Rechtskonstiuktionen werden unten in Text noch nher besprochen. 
sikohaftung reißst die zugrundeliegende Struktur der Kausalverbindungen $\mathrm{ab}$ und errichtet stattdessen eine übergreifende "Cupola" der Kollektivhaftung".

Doch anscheinend scheuen diese grundlegenden Umbauarbeiten das grelle Licht der Öfentlichkeit. Während dic Rechtsarchitekten ensig dabei sind, die neue Cupola der Risikohaftung zu konstruicren, tun sie zugleich alles, dieselbe Cupola unsichtbar zu machen. Sie verstecken sie hinter der begrifflichen Fassade der „probabilistischen Kausalität", der „Risikohaftung", des "lost value“, die alle den individuellen Charakter des Risikobeitrages betonen und die kollektive Zurechnung des Schadens verschwcigen ${ }^{4}$. George Priest, einer der führenden Konstrukteure der neuen Risikohaftung, leugnet hartnäckig, daß diese Neubauten der Haftung ein „Schwinden individueller Verantwortlichkeit bedeuten oder gar einen Erwartungswandel in Richtung unpersönlicher oder kollektiver Verantwortung". Ganz im Gegenteil,

"far from incorporating a diminished view of individual responsibility, the shift of the law's purpose toward risk control represents a vastly expanded commitment to standards of individual responsibility"s.

Gegenüber solch bemühten Versuchen, die ökologische $\mathrm{Cu}-$ pola hinter Begriffsfassaden von Individualverantwortlichkeit zu verbergen, möchte ich einige Argumente dafür vortragen, daß es Sinn macht, die Cupola dem vollen Licht der Sonne auszusetzen. Für die Weiterent wicklung grundlegender Prinzipien des Umwelthaftungsrechts dürfte es wichtig sein, genauer zu verstehen, unter welchen Umständen, in welcher Begrifflichkeit und mit welchen Folgen Gerichte und Parlamente die klassische Individualhaftung durch eine neue Kollektivhaftung crsetzen ${ }^{6}$. Insbesondere: Welche Folgen hat diese neue Rechtskonstruktion für dic Rechtsdogmatik einerseits und für das reale Handeln der Akteure andererseits? Es ist durchaus nicht von

3 Bush (1986), 1480 ff; Abraham (1987), 859 ff.

4 Kint (1981); Robinson (1985); Roscuberg(1984), $866 \mathrm{ff}$; Celli(1990), $652 \mathrm{ff}$.

5 Priest (1990), 214.

6 Bush $(1986), 1473$. 
vornherein ausgemacht, daß das Umwelthaftungrecht in der Lage ist, solche Prozesse der Risikokollektivierung ausreichend zu kontrollieren, so daß die sicheren Verluste an individueller Verantwortung tatsächlich von möglichen Gewinnen kollektiver Verantwortung aufgewogen werden. Im wesentlichen hängt dies davon ab, wie selbstorganisierende Prozesse in der sozialen Realität auf die neue Kollcktivhaftung reagicren, und davon, wic das Recht seinerseits solche Selbstorganisationsprozesse wahrnimmt und sie rechtich verfaßt ${ }^{7}$.

Ich möchte folgende vier Thesen verteidigen:

1) Wenn die Gerichte zunehmend die Kausalverbindungen zwischen Handlungen und Umweltschäden kappen, dann zichen sie nur dic praktischen Konsequenzen daraus, daß es einer Ökologie von komplex interagierenden Ursachen nicht gerecht wird, Unweltsehäden cinzelnen Entscheidungen individueller Akteure zuzurechnen. Undurchschaubare ökologische Interdependenzen drängen das Recht dazu, von einer Akteursorientierung auf cine systemische Orientierung überzusetzen. Anstatt den schuldigen Einzclakteur zu suchen, konstruiert das Haftungsrecht neuartige Risiko-Pools, ja geradezu neue formale Organisationen des Risikomanagements, die jedenfalls gegenüber manchen ökologischen Risiken angemessener erscheinen als dic traditionelle Kausalzurechnung auf Einzelaktcure.

2) Nimmt man den Kollektivcharakter der neuen Haftungsformen ernst, dann stellen sich andersartige Fragen für die Dogmatik des Haftungsrechts, die in der Individualperspektive der Akkusationskausalität nicht sichtbar werden. Die von ihr selbst geschaffenen Risiko-Pools stellen die Rechtsdogmatik vor die Herausforderung, nun auch Kriterien für deren innere Ordnung und deren Außenbeziehungen zu entwickeln. Wo sind die Grenzen eines haftungsrechtlichen Oko-Pools zu zichen? Wer ist Mitglicd? Welche mitgliedschaftlichen Pflichten und welche

7 Zum Thema Okologie und Selbstorgmistion und den Chancen, mit Mittein des Rechts okologische Selbstregulierung zu stergern, visl. die Beiträge in den Sammelband Teubner (1994). 
Rechte werden auferlegt? Unter welchen Bedingungen wird eine externe Verantwortlichkeit des Pools konstituiert? Wic wird sie nachträglich unter den Mitgliedern aufgeteilt? Kurz, wie kann man ökologische Solidarverantwortung rechtsdogmatisch formulieren?

3) Treten solche kollektiven Rechtskonstruktionen in die soziale Wirklichkeit wieder ein, so setzen sich selbstorganisierende Prozesse kollektiven Handelns in Bewegung. Dic Auswirkungen der haftungsrechtlichen Öko-Pools in der Sozialwirklichkeit sind durchaus zwiespältig. Auf der einen Seite sind negative Nebeneffekte kollektiven Handelns zu erwarten - moral hazard, free riding, Verlust von Individualanreizen. Auf der anderen Scite ist es nicht ausgeschlossen, daß sich neue kollektive Formen des Umgangs mit ökologischen Risiken herausbilden, die solche Nebeneffekte aufwiegen und sogar Anreize für ökologische Innovationen schaffen.

4) Stärke und Richtung selbstorganisierender Prozesse werden darüber entscheiden, ob das Umwelthaftungsrecht in der Lage ist, solche von ihm selbst angestoßenen Entwicklungen jedenfalls ansatzweise mit institutionellen Mitteln zu steuem. Negative Wirkungen kollektiven Handelns lassen sich zum Teil dadurch kompensicren, daß man innerhalb des Pools die Kollektiveffekte re-individualisiert. Wichtiger jedoch dürfte sein, positive Tendenzen zum kollektiven Umgang mit ökologisehen Risiken rechtlich zu verstärken. Das Recht kann die Bildung von ökologischen Kollektivakteuren so formalisieren, daß sie Umweltrisiken neu verteilen, Umweltverhalten ihrer Mitglieder steuern oder gar neue Umwelttechnologien entwickeln. Dafür dürfte es notwendig werden, die Grenze zwischen "privater" Haftung und "offentlicher Regulierung" in Formen hybrider Regulierung aufzuheben.

II. Das kollektive Element in der neuen Umwelthaftung

Juristen pflegen systematisch zu unterschärzen, welche dramatischen Änderungen eintreten, wenn unter dem Druck öko- 
logischer Schäden die juristischen Kausalketten brechen. Sie nehmen dies licber als ein bloß rechtstechnisches Problem des Beweisrechts wahr. Man müsse nur die rigiden rechtlichen Anforderungen an den Kausalbeweis ein wenig abschwächen ${ }^{8}$. Entweder ersetzt man den juristischen Vollbeweis durch einen statistischen Wahrscheinlichkeitsbeweis oder man behilft sich mit juristischen Kunstgriffen, mit dem Beweis des ersten Anscheins, mit der Umkehr der Beweislast, mit widerleglichen oder gar unwiderleglichen Vermutungen. Allenfalls räumen sie ein, daß damit der juristische Kausalitätsbegriff, der sich ja schon längst aus seiner Abhängigkeit von einer rein naturwissenschaftlichen Sichtweise gelöst hat, eine erneute Wandlung durchmacht, eine Wandlung in Richtung statistischer Verursachung und einer bloßen Risikokausalität ${ }^{9}$. Auf jeden Fall aber blieben die Wandlungen auf den Kausalitätsbegriff beschränkt und ließen das Prinzip der Individualhaftung unangetastet ${ }^{10}$.

Es scheint als könnten die Juristen den Anblick der Cupola nicht ertragen. Sie wollen nicht sehen, daR sobald das Recht das Kausalerfordernis zwischen Handlung und Schaden auch nur lockert, es mit Notwendigkeit kollektive Formen der Haftung schafft. Schon eine geringe Senkung der Anforderungen an den Kausalbeweis führt dazu, daß individuelle Akteure für Handlungen verantwortlich gemacht werden, die sie nicht begangen haben. Ihre persönliche Verantwortung ist nicht mehr ausschließlich an ihre cigenen Handlungen gebunden, die den Schaden tatsächlich veruracht haben, sondern ist dann teilweise nur noch von einer Cupola gedeckt, die sie und andere Akteure unter ihrem Dach vereint. Sie sind dann Teil einer Risikogemeinschaft, einer, sit venia verbo, Óko-Mafia, für deren Handlungen sie ciner Mithaftung nicht entrinnen können.

Dies trifft schon dann zu, wenn der volle Kausalbeweis zum bloßen Wahrscheinlichkeitsbeweis abgeschwächt wird. Genau in dem Ausmaß, in dem sich der Vollbewcis vom Wahrsehein-

8 Etwa Nicklisch (1991), $346 \mathrm{ff}$.

9 Robinson (1985); Celli (1990).

10 Pricst (1990). 
lichkeitsbewcis unterscheidet, werden sie mit einem zusätzlichen Haftungsrisiko belastet. Im Ausmaß dieser Differenz müssen sic für Handlungen anderer einstehen, über die sie kcine Kontrollc haben. Dieses Zusatzrisiko kollektiver Haftung nimmt $\%$, je mehr wir uns dem Bereich der Beweislastumkehr oder der Vermutungen nähern. Wenn der Beklagte faktisch oder rechtlich nicht den Beweis erbringen kann, daß seine Handlungen nicht kausal für den Schaden waren, dann bedcutet dies nichts anderes, als daß er für die Handlungen anderer einstehen muk.

Wir haben in diesen Fällen cine asymmetrische Kollcktivverantwortung vor uns, eine Art horizontaler vikarische Haftung für fremdes Handeln. Asymmetrisch ist die Haftung insofern, als nur cin Mitglied des Haftungskollektivs, der Beklagte, feststeht, während andere Mitglieder im Nebel der ungeklärten Kausalverhältnisse unsichtbar bleiben. Horizontal ist diese Haftung im Unterschied zur vertikalen Haftung in hierarchischen Organisationen, in denen die Organisationsspitze für das Handeln cinfacher Organisationsmitglicder verantwortlich gemacht wird.

Symmetrisch wird dic vikarische Haftung in den Fällen der Multikausalität, in denen eine ganze Gruppe von potentiellen Schädigern haftet, ohne daß der exakte Kausalbeweis geführt werden konnte, ebenso wie in den neuen haftungsrechtlichen Erfindungen, der market share liability, der SuperfundHaftung, und ganz allgemein in Fällen der bloßen Risikohaftung. In der Sache macht die schicre Mitgliedschaft in einer Gruppe von Risikoträgern für fremdes Individualhandeln verantwortlich. Natürlich kann man dies dennoch „Risikohaftung", also Verantwortung für den cinzelnen Risikobcitrag nennen und damit das individuelle Element betonen. Im Strafrecht macht es auch vom Standpunkt ciner Individualhaftung Sinn, in manchen Fällen schon die Setzung ciner Gefahr und nicht erst den Eintritt cines Erfolges mit Sanktionen zu belegen. Aber zivilrechtlich geht es primär darum, einen tatsächlich eingetretenen Schaden zu kompensieren. Hier ist die Frage, nicht wer für cin - im übrigen häufig erlaubtes - Risiko mit Sanktionen zu 
belegen ist, sondern wer für die eingetretene Schadensfolge aufzukommen hat. Mag auch das Risiko individuell zurechenbar sein, das eigentlich relevante Elcment, der cingetretene Schaden, ist nur kollektiv zurechenbar. Wer Mitglied ist, zahlt. Nicht dic Handlung, sondern die Mitgliedschaft macht für den Schaden haftbar.

Was Priest \& Co. mit dem Euphemismus cines „vastly expanded commitment to standards of individual responsibility" belegen, ist also in Wahrheit nur der verzweifelte Versuch von eingeschworenen Individualisten, post factum mit den perversen Effekten ihrer eigenen Manipulation am Kausalitätsbegriff fertigzuwerden. Ihre Sünde wider den Geist des methodologischen Individualismus bestcht darin, den statistischen Kausalbeweis als Haftungsgrundlage zugelassen zu haben ". Der Iluch dieser bösen Tat ist es, daß sie cin Haftungskollektiv gebiert, das im Prinzip keine Grenzen kennt. Deshalb sollte man nicht länger vergeblich versuchen, diesen Effekt herunterzuspielen, sondern sich der radikalen Konsequenz stellen, daB unter der Herrschaft der Risikobeitragshaftung jede Handlung in der Gesellschaft - und jede Unterlassung! - zum Risiko beiträgt ${ }^{12}$. Dann erst wird mit aller Deutlichkeit sichtbar, daß man innerhalb des Groß-Risiko-Pools der Gesamtgesellschaft künstlich neue Grenzen ziehen muR, die mit einer gewissen Willkür kleinere Risiko-Pools schaffen, und dal man mit ähnlicher Willkür innerhalb dieser Pools Verantwortichkeit re-individualisieren muß. Genau dies tun dic Rechtsarchitekten der Cupola: Zuerst kollektivieren sie die Haftung und dann versuchen sic, dic Konsequenzen ihres eigenen Handelns dadurch wieder rückgängig zu machen, daß sie die Kollektivhaftung aut die Mitglieder verteilen. Zunächst definicren sic Haftungsregeln für das Kollektiv und dann definieren sie interne Organisationsregeln für die interne Reallokation der Haftung, indem sie die Individualanteile an der Kollektivhaftung bestimmen.

11 Bush (1986), 1493.

12 So selbst Priest (1990: 215), der dann aber hinter seine cigene Position zurückfallt. 
Wie also soll man solch dramatische Veränderungen im Umwelthaftungsrecht verstehen? Offensichtlich lassen sie sich nicht auf Wandlungen nur des Kausalitätsbegriffes reduzieren. Sie führen vielmehr auf die grundsätzlichere Frage,

"ob die Zurchnung auf individuelles Fntscheiden (sei cs rational, sei es intuitiv, gewohnheitsmäligy usw.) uberhaupt noch haltbar ist. Oder ob man nicht unabhängig divon cinen strikt soziologisehen Ansatz ausprobieren sollte, der das Phinomen Risiko nur am Sinn von Konmunikationen erfalist" ${ }^{3}$.

Ein solcher Ansatz würde statt individueller Akteure als risikocrzeugende Einheiten Handlungssysteme identifizieren, Kommunikationsnetzwerke und nicht Einzelmenschen. Er würde sich auf selbstorganisierende Prozesse der Risikokommunikation konzentrieren und nicht auf individuelle Motive und Präferenzen, individuelle Risikowahrnehmungen, individuelle Entscheidungen und individuelle Verantwortlichkeit. Ein solcher soziologischer Ansatz, würde damit rechnen, $\mathrm{daB}$ in der heutigen Gesellschaft Risiko- und Verantwortungszurechnung auf Kommunikationen auch dann stattfindet, wenn sich gar keine individuelle Entscheidung, geschwcige denn ein Kausalnexus zwischen ihr und dem Ökoschaden identifizieren läßt.

Die Undurchschaubarkeit von ökologischen Kausalverknüpfungen ist der Grund dafür, daß die grundlegenden Annahmen des Haftungsrechts in eine tiefe Krise geraten sind. Während die Ursachen und Symptome dicser Krise schon ausrcichend analysiert sind, ist es heute noch ziemlich unklar, in welche Richtung sich die Umwelthaftung entwickeln wird.

Als Ursachen der Krise werden im wesentlichen drei Komplexe genannt, welche die individuclle Kausalzurechnung in Schwierigkeiten bringen ${ }^{14}$. Erstens der sogenannte Schmetterlingseffekt, wonach kleine technologische Änderungen nur allmählich akkumulieren, dann aber plötzlich katastrophale Änderungen auslösen können. Zweitens Probleme der Interferenz technologiseher Neuerungen. Drittens hochunwahr-

13 Luhmann (199), 13 .

14 Bechmam (1900), $128 \mathrm{ff}$ Bedman (1991), $222 \mathrm{ff}$, Wagner (1990), $27 \mathrm{ff}$ 
scheinliche Koinzidenzen, wenn zwei oder mehrere Kausalketten in nicht vorhersehbare Weise zusammenlaufen.

Symptome der Krise des Haftungsrechts sind auf Spannungen z.wischen der neuartigen Risikostruktur in der Ökologie und den Grundbegriffen des Haftungsrechts zurückzuführen ${ }^{15}$ :

1) Wie soll man noch einen individuellen ,Schädiger", die einzelne "Handlung", den abgrenzbaren "Schaden" feststellen, wenn Langzeitschäden zu beurteilen sind, in denen mehrere Kausalketten zusammenlaufen?

2) Wie soll man eine kausale Verbindung zwischen Handlung und Schaden isolieren, wenn multiple oder zirkuläre Kausalität vorliegt?

3) Wie lassen sich potentiell gefährliche Handlungen sinnvoll cingrenzen, wenn alles soziale Handeln risikobehaftet ist?

4) Wie kann man Schadensopfer identifizieren, wenn sic nur eine amorphe Masse darstellen (Umweltschäden, zukünftige Generationen)?

Wenn dies heute weitgehend konsentierte Ursachen und Symptome der Krise sind, in welche Richtung wird das Unwelthaftungsrecht von ihnen gedrängt? Hat es schon die absoluten Grenzen seiner Wandlungsfähigkeit erreicht, die von den Komponenten Akteur-Kausalität-Schaden definiert sind? Oder ist das Umweltrecht in der Lage, systemische und kollektive Elemente in die Individualhaftung $z u$ inkorporieren?

Hierzu erscheint es erforderlich, das kollektive Element, das in das Umwelthafrungsrecht eindringt, näher zu bestimmen. Offensichtlich haben wir es hier nicht nur mit Kollektivierung des Haftungsrecht in dem uns vertrauten Sinne zu tun, daß die Haftung von einer Zurechnung auf höchstpersönliche Eigenschaften einer "Person“ umgestellt wird auf eine Zurechnung auf eine standardisierte "Rolle“. Vichmehr ist die Zurechnung auf individuelle Positionen als solche zunchmenden Zweifeln ausgesetzt. Doch ist es auch zu einfach, hier wic Bush (1986)

15 Rabin (1987), $27 \mathrm{ff}$; Brüggemeicr (1991), $297 \mathrm{ff}$; Brüggencicr (1994); 1 uhmann (1991), 99 f; Schmidt (1991), 378 ff. 
und Abraham (1987) nur von einer allgemeinen Tendenz zu kollektiver Haftung zu sprechen ${ }^{16}$.

Ebensowenig einschlägig sind die Standardfälle kollcktiver Haftung - Haftung für fremdes Handeln $(\$ \$ 31,278,831$ BGB) - in denen Manager in den höheren Rängen einer Organisation oder die Organisation selbst für die Handlungen von Organisationsmitgliedern verantwortich gemacht werden. In diesen Fällen zielt die „policy“ des Haftungsrechts nicht nur darauf, den Opfern bessere Ausgleichsmöglichkeiten zu verschaffen, sondern besonders darauf, dic Risikowahrnehmung ganzer Organisationen statt der individueller Akteure zu verändern ${ }^{17}$. Aber in unseren Fällen kollektiver Umwelthaftung gibt es keine schon existierende Organisation, keine bestehende Hicrarchie, kein schon definicrter Kollektivakteur, der als Zurechnungsendpunkt dienen könnte.

Auch nicht vergleichbar sind Situationen des ,piercing the corporate veil", des Haftungsdurchgriffs im Konzernrecht oder im Vertragsecht, in denen die traditioncllen Grenzen der Haftungsbeschränkung von Korporationen oder des Relativitätsprinzips des Vertrages überschritten werden ${ }^{18}$. Denn auch in diesen Fällen bezicht sich das Recht auf schon existierende Kollektivakteure oder auf relativ stabile vertragliche Arrangements und macht die Gesamtorganisation verantwortich für das Handeln ihrer Mitglieder. Zwar redefiniert das Recht die kollektiven Einheiten nach seinen Haftungspolicies, oft genug gegen die Absichten seiner Gründer. Aber solche Ausdchnungen individueller Haftung können doch die Grenzen eines schon vorweg existierenden kollektiven Arrangements nicht überschreiten.

Anders ist die Lage, wenn das noue Recht der Risikohaftung cinfach ganze Produktmärkte finanzicll haften läßt oder wenn es die sogenannten "bubbles" der Luftverschmutzer oder ganze umweltverseuchte Regionen verantwortlich macht. Hier verläßst es endgültig dic Akteursperspektive, weil es nicht einmal

16 Kondyen (1991), 105.

17 Brüggemeier (1994); Hofstetter (1994).

18 vel. I Tofstetter (1994); Adams/Brownsword (1990). 
mehr nach Kollektivakteuren sucht, und konzentriert sich auf Risikokommunikation als solche. Riskante Kommunikationen entstehen innerhalb sozialer Konfigurationen, die sich nicht als formale Organisationen identifizieren lassen. Und das Recht rechnet die Verantwortung der Risikokommunikation direkt zu - einem Produktmarkt, ciner kontaminicrten Region, ciner "Luftblase“. Es macht also Handlungssysteme selbst verantwortlich, ohne sich um deren Eigenschaft als oryanisierte Willensbildungseinheiten zu kümmern. Der entscheidende Unterschied der neuen Risikohaftung zu bckannten Formen der Organisationshaftung besteht demnach darin, $\mathrm{da} 3$ das Haftungsrecht sich nicht mebr anf bestehende korporative Arrangements bezieht, sondern selbst newartige Risikonetzwerke definiert. Es versucht nicht bloß, die Risikowahrnehmung existierender Kollektivaktcure zu beeinflussen, die als Resultat von Organisationsentscheidungen schon in der sozialen Welt sind. Vialmehr sucht es, unkoordinierte Risikokommunikation in cinem diffusen sozialen Feld dadurch zu becinflussen, dab es diesem Feld ein kritisches Risikomaß zuschreibt. Und sobald individuelle oder kollektive Akteure in einen solchen kommunikativen Raum geraten, worden sie Zwangsmitglieder eines solchen Risikopools - also nicht kraft privatautonomer Entscheidung, sondern kraft autoritativer Anordnung des staatlichen Rechts. Sie sind dann in der Kollektivhaftung ohne Rücksicht auf ihren Willen und ohne Rücksicht auf den Kausalzusammenhang $\angle$ wischen den von thnen individuell verantwortbaren Handlungen und dem eingetretenen Umweltschaden.

In manchen Fällen geht das Umwelthaftungsrecht noch einen Schritt weiter. Modernes Umweltrecht scheint von Bismarcks Zwangsgenossenschaften der Sozialversicherung inspiriert, wenn es - wie es in der deutschen Diskussion zu den Umweltgenossenschaften vorgeschlagen wird ${ }^{19}$ und wie es in der amerikanischen Superfund-Haftung tatsächlich geschieht ${ }^{20}$ neue Risikoorganisationen gründet. Hier werden nicht nur cin-

19 Kinkel (1989), 297; Rebbinder (1989), 161; Wagner (1990), 5211 .

20 Stewart (199)!). 
fache Haftungspools eingerichtet, sondern es werden Zwangsorganisationen mit kollektiver Handlungsfähigkeit ausgestattet. Die Verantwortung der Pools bestcht nicht nur für den finanziellen Ausgleich ökologischer Schäden, sondern für den kollektiven Umgang mit den Umweltrisiken selbst. Es werden neue kollektive Einheiten des Risikomanagements geschaffen, in denen kollektive Haftung mit aktiver Regulicrung kollektiver Innovation zusammenfällt ${ }^{2 !}$.

Angesichts solch weitreichender Veränderungen versteht man nun besser, wieso manche Haftungsrechtler emphatisch vor jeglicher Manipulation des Kausalzusammenhangs warnen ${ }^{22}$. Was so harmlos begann als eine Forderung der Billigkeit - im Falle komplexer Kausalzusammenhänge die Opfer nicht ohne Schadensausgleich zu lassen - stellt sich nun als eine grundsätzliche Unwandlung von Individualhaftung in kollektive Risikopoolung heraus. Dic Manipulationen am Kausalzusammenhang erscheinen ihnen als ein „overshooting":

"D.s Dilenma jeylicher Fortentwicklung im Bercich der Kausalität besteht allerdings darin, dals ein Uberdrehen der Kausalitätsschraube zu einer Übermakhaftung fülirt, die nit falsehen Präventionsanreven verbunden ist und dic Ressourcenallokation verfïlsche, da derjenige mit Kosten belastet wird, der den Sthaden niche verursach hat und ihn deshalb auch nicht vermediden kann" ${ }^{23}$.

Mit diesem zunächst plausiblen Argument übersehen sie jedoch zwei wesentliche Gesichtspunkte. Erstens wäre es nicht nur unbillig, sondern zugleich ineffizient, wenn in den Fällen undurchschaubarer Kausalzusammenhänge die Opfer ohne Ausgleich blieben. Denn dann bestünden nicht nur schwache, sondern gar keine Präventionsanreize und die Ressourcenallokation wäre noch mehr verfälscht ${ }^{24}$. Denn in diesen Fällen steht man gar nicht vor der Wahl kollektive versus individuelle Haftung, sondern, da der fehlende Kausalnexus individuelle Haftung über-

21 Rase-Ackeman (1990), 746.

22 Lpstein (1985), 1377; Medicus (1986), 781, 785; Abraham (1987), 898,

23 Rehbinder (1989), 157; vil. auch Assmann (1988), 111.

24 So anch Rehbinder (1989), 157. 
haupt konterkariert, kollektive Haftung oder gar keine Haftung.

Zweitens treffen sie zwar genau den kritischen Punkt, wenn sie argumentieren, daß individuelle Akteure für Schäden verantwortlich gemacht werden, die sie nicht vcrursacht haben. Doch übersehen sie dic Cupola als verantwortlichen Kollektivakteur. Sie unterschätzen systematisch das Potential kollektiver Handlungs- und Verant wortungszurechnung. Dies hängt eng damit zusammen, daßs sie unter den Prämissen des methodologischen Individualismus selbst klargeschnittene Kollektivakteure nicht mehr wahrnehmen, sondern in einen bloßen Nexus von Individualverträgen autlösen. Deshalb halten sie an individueller Zurechnung auch in Situationen fest, von denen sie wissen, daß es nicht nur inadäquat, sondern von vornhercin ausgeschlossen ist, kausale Verbindungen herzustellen. Sobald man aber einräumt, daß in solchen Situationen Risiko, Handlung, Kausalität und Verantwortung Risiko-Pools zugerechnet werden können, die das Recht selbst durch "Fiat" geschaffen hat, dann sieht man auch, dał das Recht dann in der Tat dic Handlungseinheiten mit Kosten belastet, die den Schaden tatsächlich verursacht haben und die auch dazu beitragen können, den Schaden zu vermeiden. Freilich muk man dann Anreize und Ressourcenallokation umdenken, weil man jetzt weder mit Individuen noch mit schon existierenden Kollektiven zu tun hat, sondern mit neugeschaffenen Risikokollektiven.

\section{Die Umrisse der Cupola}

Wenn die Architekten des neuen Haftungsrechts die Kausalverstrebungen der individuellen Verantwortung durch die Cupola kollektiver Haftung ersetzen, dann stellen sich ihnen cine ganze Reihe neuartiger Konstruktionsprobleme. Wie weit kann man die Kuppel ausdehnen, ohne daß das ganze Bauwerk zusammenbricht? Welche Personenkreise sollen unter dem Dach der Cupola als Mirglieder erfaßt werden? Welche Pflichten legt die Mitgliedschaft in der Cupola den Mitgliedern auf? Wie soll 
die Gesamthaftung der Cupola unter den Mitglicdern aufgeteilt werden?

Vorrangige Frage für dic Rechtsdogmatik ist, wie der Risikopool zu identifizieren ist. Wenn dic Kausalverbindungen abgebrochen sind, dann wird es außerordentlich schwierig, vernünftige Grenzen der Haftung für risikoerzeugende Aktivitäten zu finden. Wie schon gesagt, potentiell trägt jede soziale Kommunikation zum ökologischen Risiko bei. "Almost every human action will increase the probability of loss in all contexts. It follows, thus, that under modern conception of risk, no action is ever truly innocent" ${ }^{25}$. Und dic cigentliche Frage heißt jetzt, wic weit man die Durchsetzung von Haftungsstandards treiben will, die nur auf Schadenswahrscheinlichkeiten gestützt $\operatorname{sind}^{26}$.

Gesucht: Kollektivakteur - so könnte man die Aktivitäten der Gerichte umschreiben, die, nachdem der Verlust der Individualakteure cinmal feststand, cinc neue Lösung für multiple Kausalität suchten. Im Unterschied zu den Versuchen, das Problem zu einer rein technischen Bewcisfrage zu machen, waren die cher anspruchsvollen dogmatischen Anstrengungen darauf gerichtet, praktikable Kriterien für ein handlungsfähiges Kollektiv, eine zweckgerichtete Organisation oder wenigstens cine lose Form planvoller Zusammenarbcit zu entwickeln, mit deren Hilfe man kollektive Haftung begründen könnte.

In den USA war "concerted action“ der einschlägige deliktsrechtliche Begriff, wonach gemeinschaftliche Haftung auferlegt wurde,

,all those who in pusuance of a common plan or design to commit a tortious act, actively take part in it, or further it by coperation and request, or who lend aid or encouragment to the wrongdoer, or ratify and adopt the wrongdoer's acts done for their benchit: 2 .

Diese Doktrin identifiziert cin Kollektiv über bestimmte Eigenschaften der beteiligten individuellen Aktcure (Kooperationsabsicht) und über bestimmte Eigenschaften ihrer Handlun-

25 Priest (1990), 215.

26 Robinson (1985), 796 .

27 Prosser/Keeton (1984), 323 . 
gen (Verwobenheit). Sic lehnt sich so eng wie möglich an das ursprüngliche Handlungsmodell an: "the act of one is the act of all, and liability for all that is done is visited on each" ${ }^{28}$. Ökologische Risiken jedoch beugen sich nicht der Logik der Kooperation und gehorchen nicht der Doktrin der ,concerted action". Ausdrückliche Vereinbarungen, dic Umwelt zu schädigen, sind nicht gerade häufig, klammheimlicher Konsens ist schwer zu beweisen. Dic ganze Konstruktion scheint auf die typische Konstellation multipler Kausalität nicht zu passen.

Dann versuchten die Gerichte es mit der sogenannten „enterprise liability", indem sie die Anforderungen an bewuites, geplantes und zweckgerichtetes Zusammenarbeiten noch weiter zurückschraubten ${ }^{29}$. Im Fall Hall ws. DuPont gab sich das Gericht schon damit zufrieden, ein blokes ,joint enterprise“ zwischen ansonsten unabhängigen Akteuren zu identifizicren, sofern nur cine gemeinsame Risikokontrolle in Form gemeinsamer Sicherheitsstandards und der lunktionsdelegation an einen Industricverband bestand ${ }^{30}$. Eine förmliche, joint venture braucht nicht nachgewiesen zu werden, es genügen sehon Beweise über gemeinsame Forschung, gemeinsame Produktprüfungen und gemeinsame Lobbyaktivitäten ${ }^{31}$. Als Minimalerfordernis für diese Form der Kollektivhaftung gilt ein unzureichender industrieweiter Sicherheitsstandard für die Güterherstellung ${ }^{32}$. Doch auch diese Haftungsform ist der typischen Risikostruktur wenig angemessen und sollte sich selbst als kontra-produktiv herausstellen. Sie kann nicht Formen des bloßen Parallelverhaltens der Hersteller erfassen ${ }^{33}$. Ja, sie privilegiert das umweltschädigende Parallelverhalten gegenüber Versuchen, das Schadensrisiko durch kollektive Anstrengungen zu begrenzen. Diese werden mit „enterprise liability" belegt,

28 Prosser \& Keeton (1984), 346.

29 Sheiner (1978), 995-1006; Podgers (1980), 827.

30 Hall ×. DuPont, 345 F. Supp. 353 (F.D.N.Y. 1972), at 375 6.

31 Comor v. Grand Western Savings \& Lam Association (1968)69 Cal. $2 \mathrm{~d}$ 850.

32 Sheiner (1978), 995.

33 Bush (1986), 1483; Spitz (1990), 626. 
während das rein individuelle Parallelverhalten ohne jeden Versuch der kollektiven Risikokontrolle haftungsfrei bleibt. „Enterprise liability" hat also die nichtbeabsichtigte Nebenfolge, negative Anrcize für industrieweite Zusammenarbeit in der Risikokontrolle zu setzen.

Dann erscheint es nur folgerichtig, die vergebliche Suche nach dem Kollcktivakteur aufzugeben und den Markt selbst als die Haftungscinheit zu wählen, und dies obwohl der Markt gerade keine kooperativen, sondern nur kompetitive Strukturen aufwcist. Genau dies geschah mit Hilfe der berühmten „market share liability“34. Damit werden endgültig alle Versuche, jedenfalls Spuren einer übergreifenden Organisation, sei es eines Unternehmens, einer Unternehmensgruppe, sei es eines Kooperationsnetzes, letztlich aufgegeben und durch die Suche nach den "relevanten Markt" ersetz.t.

Sobald cin Akteur in cinen Markt eintritt, wird er für die Umweltrisiken, die im Markt entstehen, verantwortlich gemacht. Diese kühne Idee steht im klaren Widerspruch zu traditionellen Grundsätzen der Kollektivhaftung ${ }^{35}$. Kollektivhaftung setzt Zusammenarbeit, gemeinsame Aktivitäten, gemeinsame Kontrolle voraus ${ }^{36}$. Der Konkurrenzmarkt aber ist das genaue Gegentcil eines gemeinsamen Unternehmens als plausible Basis einer Kollektivhaftung. Wie kann ich für die Handlung meiner Konkurrenten haftbar gemacht werden, wenn ich mit ihnen nicht zusammenarbeite, im Gegenteil alles tue, um sie zu bekämpfen, und wenn ich zudem keinerlei Kontrollmöglichkeiten über ihre Handlungen besitze. Das widerspricht diametral auch dem Laienverständnis, wonach "companies are liable only for the harm their own product docs, not for their rivals' damage “37. Und auch dic anteilmässige Begrenzung der "market sharc liability ${ }^{\text {"x }}$ auf meinen Marktanteil verändert die Sachlage nicht. Denn auch mit der Anteilshaftung werde ich

34 Dazu Abraham (1987), $861 \mathrm{ff}$.

35 Bush (1986), 1477.

36 French $(1982),(1984)$.

37 The Leconomist, Itel. 29, 1992, 16. 
für schädigende Handlungen anderer verantwortlich gemacht. Die Anteilsbegrenzung nimmt nur die ohnchin später fällige individuelle Schadensaufteilung vorweg, ohne dic prinzipielle „Ungerechtigkeit“ ciner Markthaftung zu beseitigen.

Die deutsche Rechtsentwicklung kennt durchaus ähnliche Tendenzen mit ähnlich dilemmatischen Ergebnissen ${ }^{38}$. Das BGB kennt die gesamtschuldnerische Haftung von Mittätern, wenn jeder cinzelne Beteiligte den ganzen Schaden hätte verursachen können und die schadensverursachende Handlung gemeinschaftich begangen wurde (\$830 I 2 BGB). Ursprünglich verlangte die Rechtsprechung cine echte Kooperation zwischen den Beteiligten, zumindest aber ein gemeinsames Bewußtsein in dem Sinne, daß jedem Beteiligten die gefährlichen Handlungen der anderen Beteiligten bewußt gewesen sein mußten. Aber die Anforderungen an diese Verbindungen wurden immer mehr gelockert, so daß heute eine gewisse „räumliche oder zeitliche Einheit" genügt. Der BGH geht sogar so weit, die Beweislast umzukehren, wenn dic Verbindung zweicr Kausalketten dic Feststellung individueller Verursachung unmöglich macht ${ }^{39}-$ ein Ergebnis, daß von akademischen Kommentatoren als prinzipienlose Billigkeitsrechtsprechung kritisiert wird ${ }^{40}$. Während der Vorarbeiten für das neue Umwelthaftungsgesetz ${ }^{41}$ wurde ernsthaft erörtert, in bestimmten ökologischen Schlüsselbereichen das Erfordernis der „alternativen Kausalität" aufzugeben und den Verschmutzer bereits dann gesamtschuldnerisch haften zu lassen, wenn er zu dem ökologischen Risiko beigetragen hatte. Die endgültige Fassung des Umwelthafungsgesetzes ist allerdings wesentlich enger: $\$ 6 \mathrm{UmweltHG}$ begründet eine Vermutung für die Kausalität cines Anlagenbetreibers unter bestimmten Umständen, und $\$ 7$ UmweltHG erweitert diese Vermutung für den Fall mehrerer Betreiber.

38 Medicus (1986); Assmann (1988); Brügsemeicr (1991); Köndgen (1991).

39 BGHZ, 66, 70 - „Stcinbruch" -1976.

40 Köndgen (1991), 101 t.

41 Geserz über die Umwdthaftung vom 10.12.1990, BGB1.1, S. 2634. 
Die deutschen dogmatischen Entwicklungen führen zu etwas anderen Resultaten als ihre amerikanischen Gegenstücke. Etwas haben sie allerdings gemeinsam: Ich behaupte, daß auch das deutsche Haftungsrecht ein Handlungs- und Haftungskollektiv in Fällen nicht-kooperativen Verhaltens neu konstituiert. Die Gerichte fahnden nicht mehr nach einem vorhergehenden bewußten Zusammenwirken als Voraussetzung kollektiver Haftung. Sie haben die Verbindung zwischen rechtlicher Kollektivhaftung und dem Tatbestand gemeinschaftlichen Verhaltens, der Existenz ciner festgefügten Gruppe oder gar eines ausgewachsenen korporativen Akteurs in der sozialen Realitär gekappt. Sie suchen nicht mehr nach Kollektiven in der sozialen Welt, sondern erschaffen das Kollektiv schlicht durch Richterspruch.

Diese Innovation löst ein Problem und schafft gleichzeitig cin neues. Wenn das Kollektiv nicht in der sozialen Realität vorgegeben ist, worauf sich das Recht bei der Zurechnung kollektiver Verantwortlichkeit beziehen könnte, welchem Grundsatz. folgt dann die künstlich-autoritative Schaffung eines Risikopools durch das Reche? Die amerikanische Erfahrung würde für den Markt als die risikocrzeugende Einheit sprechen. Aber welcher ist der ,relevante Markt": der lokale, der regionale, der nationale, der globale Markt? Ist die Haftung nach Marktanteilen angemessen, wenn der Schaden geographisch weit entfernt von einem industricllen Verschmutzer in diesem Markt auftritt? Und warum Markt- und nicht Branchenhaftung? In dem berühmt-berüchtigten DES-Fall war dic Wahl des nationalen Marktes der USA eine überzeugende Lösung, um die gefährlichen medizinischen Produkte abzugrenzen, aber ist der Markt überhaupt ein verallgemeinerungsfähiges Kriterium für Fälle ökologischer Haftung?

Die deutsche Lösung arbeitet mit den Kriterien einer abstrakten „räumlichen oder zeitlichen Einheit riskanter Betätigungen“ oder eines ähnlich abstrakten ,unaufklärbaren Zusammenwirkens von Ursachenzusammenhängen“". Beide Kriterien leiden unter der üblichen Schwäche deutscher Abstraktionen: theore- 
tisch vielleicht einleuchtend, aber zu unbestimmt und zu allgemein, um praktisch brauchbar zu sein.

Ich schlage vor, den Risikopool nicht mehr durch soziale Kriterien (kooperative oder korporative Strukturen) zu bestimmen zu versuchen. Er sollte vielmehr als ökologischer Problembereich definiert werden, dessen Grenzen durch dic Eignung für kollektives Risikomanagement zu bestimmen sind. Letztlich entscheidend sind weder ökologische Kausalzusammenhänge noch vorgegebene kooperative Strukturen - so wichtig beides auch sein mag - , sondern zentrales Kriterium sollte die Fähigkeit des Pools zum Risikomanagement sein. Zugegeben, das ist "opportunistische" Zurcchnung ${ }^{42}$, in diesem Fall nicht kausaler, sondern kollektiver Art. Für den Zweck der kollektiven Haftung identifiziert das Recht konkrete ökologische „Risikogebiete" (einen See, einen Fluß, eine Landschaft, einen Luftraum, cine Produktionskettc, einen ökologischen Kreislauf) mit dem Hintergedanken, cin soziales Gebilde zu schaffen, das diese Risiken beherrschen kann oder das wenigstens zur Schadensabwicklung herangezogen werden kann.

Unter Risikomanagement verstehe ich hier erstens die Abwicklung bereits entstandener Schäden. Das Recht gestaltet das Kollcktiv so, daß in Fällen mehrfacher Kausalität die Schäden ausgeglichen werden können, indem es cinen ausreichenden finanziellen Pool schafft, der die Verluste abdeckt und das Risiko verteilt ("deep pocket", ,risk spreading ${ }^{\text {") }}$. Zweitens - und das ist womöglich wichtiger - bedeutet Risikomanagement die kollektive Steucrung zukünftigen Verhaltens ${ }^{43}$. Das Recht zicht die Konturen des Risikopools derart, daß eine realistische $\mathrm{Ba}$ sis für eine aktive und gemeinsame Prävention von Risiken in Gebieten geschaffen wird, wo ökologische Probleme konzentriert sind. Unter beiden Gesichtspunkten isolicrt das Recht den sozialen Bereich kollektiver Verantwortlichkeit unter Heranziehung ökologischer, geographischer und gesellschaftlicher Kriterien, so daß sich eine funktionsfähige gemeinsame Techno-

42 I.uhmann (1991), 47, und nich Williamson (1985), 129.

43 Bush (1986), $1553 \mathrm{fl}$. 
logie zur Bewältigung ökologischer Risiken entwickeln kann. In Konfliktfällen könnten sogar ökologische Zusammenhänge übergangen werden, wenn sich dennoch vernünftige Grenzen für cin solches soziales Gebilde finden lassen.

Zugegebenermaßen bietet diese Formel keine vergleichbar scharfe begriffliche Abgrenzung des Kollektivs wic sie dic herkömmliche Suche nach vorgegebenen hierarchischen oder kooperativen Gebilden in der Sozialwirklichkeit anbicten konnte. Es handelt sich eher um einen "policy-mix", der über eine vernünftige Zusammenfassung der Risiken entscheidet. Dic Situation gleicht dem „strategic policy-mix“, den Versicherungen benutzen, wenn sic die Versicherten in verschicdene Risikokategorien einteilen ${ }^{4+}$. Die Formel ist deutlich umfassender als dic von der amerikanischen Doktrin bevorzugte Konzentration auf einen Markt und die jewciligen Risikobeiträge, andererseits aber enger als die deutsche Abstraktion ciner rïumlich-\%citlichen Einheit, die auf Kriterien zur Bestimmung dieser Einheit verzichtet. Die Formel erlaubt es, die ganze Bandbreite möglicher ökologischer Risikopoole $z u$ identifizieren, die kollektive Haftung auslösen. Mit ihr lassen sich nicht nur bestimmte Produktmärkte, sondern auch ökologische Ketten, kontaminierte Areale, vergiftete Seen und Flüsse, "pollution bubbles" und andere ökologische Problemgebiete crfassen. Ausschlaggebendes Kriterium sollte aber meiner Ansicht nach die Möglichkeit eines ökologischen Risikomanagements sein.

Im Lichte dieser Formel wäre "market share liablity" nur eine unter mehreren Möglichkeiten, cin ökologisches Problemgebiet nach seiner Eignung für Risikomanagement auszusuchen. Im Fall gefährlicher Produkte, die auf cinem bestimmten Markt vertricben werden, liegt es in der Tat nahe, das ökologische Risikogebict anhand dieses Marktes zu bestimmen:

"... the industry rather than the individual manufacturer should be the focal point for liability because it can best allocate risks, distribute costs, and take preventive meisures ${ }^{45}$.

44 "Riste classification", Abraham (1988), 949; Eubank (1991), 194.

45 Sheiner (1978), 1002-4. 
Das Folgeproblem, wie der ,appropriate market ${ }^{46}$ zu bestimmen ist - im Hinblick auf seine geographische Ausdehnung und auf die Identität des Produkts -, sollte nicht nur anhand seiner ökonomischen Eigenschaften (Substituierbarkeit des Produkts, Marketingstrategien, Intensität der Transaktionen) gelöst werden, sondern ganz offen mit Hilfe der Kriterien eines ökologischen Risikomanagements. Häufig ist es durchaus unklar, ob der "appropriate market" der lokale, der nationale oder der globale Markt ist. Die Entscheidung sollte dann nicht ohne Rücksicht auf folgende Uberlegungen getroffen werden: Ist die Bestimmung des Marktes weit genug, um die Schäden finanziell ausglcichen zu können? Wird die Bestimmung des Marktes zu einer akzeptablen Risikoverteilung führen? Ist sie andererseits eng genug, um den beteiligten Akteuren die Möglichkeit zur Kooperation und zur Entwicklung einer dezentralisierten Risikokontrolle zu cröffnen? Gibt es realistische Möglichkeit kollektiven Risikomanagements?

Im Fall Hall v. Dupont spicten diese Überlegungen eine erkennbare Rolle. Das Gericht betonte, seine Regel sei nur anwendbar auf

"... industries composed of a small number of units. What would be fair and feasible with regard to an industry of five to ten producers might be manifestly unreasonable if appliced to a decentralized industry composed of thousinds of small producersit?

Die Haftung nach Marktanteilen löst eine partielle horizontale Integration der betroffenen Unternchmen aus. Das Haftungsrecht schafft sozusagen ,joint ventures" für kollektives Risikomanagement in einem bestimmten Markt. Das ist sinnvoll, wenn - wie im DES-Fall - cin einheitlicher Markt für die gefährlichen Produkte existiert. Unter anderen Umständen bestehen allerdings andersartige ökologische Risiken. In einer ökologischen Kette können die typischen Gefahren nur crkannt werden, wenn man die verschiedenen Produktions- und Absatz-

46 Spitz (1990),619 ff.

47345 f. Supp. 353 (E.D.N.Y. 1972) at p. 378. 
stufen betrachtet: Bezug von Rohstoffen, Produktion, Vertrieb, Verbrauch und Abfallentsorgung. In diesen Fällen ist anstatt einer horizontalen cher eine particlle vertikale Integration angezcigt. Das Haftungsrecht sollte eine vertikale Haftungskette schaffen, die dic Herausbildung neuer Formen vertikalen Risikomanagements entlang der verschiedenen Phasen des Produktionsprozesses stimulieren könnte. In Japan und Deutschland wurden bereits Erfahrungen mit ökologischen Vereinbarungen zwischen den Firmen einer Produktionskette gesammelt ${ }^{48}$. Das Haftungsrecht sollte nicht zögern, durch die Drohung mit seinen drastischen finanziellen Sanktionen solche Vereinbarungen zu erleichtern.

Dem amerikanischen "Superfund“ liegt wiederum ein anderes Prinzip zugrunde ${ }^{49}$. Hier werden nicht sozio-ökonomische Konfigurationen haftbar gemacht - weder formale Organisationen wie bei der „enterprise liability“, noch Märkte wie bei der Haftung nach Marktanteilen, noch vertikale Produktionsketten. Statt dessen definiert das Recht soziale Einheiten nach geographischen Kriterien. Kontaminierte Landschaften sind die neuen ökologischen Problembereiche, zu denen bestimmte Akteure in einer engen Bezichung stehen. Das Recht schafft ein Zwangskollektiv dieser reichlich heterogenen Akteure - Landeigentümer, Produzenten gefährlicher Stoffe, Transportunternehmer und Manager des kontaminierten Grundstücks -, die es gesamtschuldnerisch für die Schäden und für die Kosten der Wiederherstellung haftbar macht. Die „Einheit“ des Risikomanagements ist ein bestimmtes soziales Arrangement, das um einen geographischen Risikobercich herum zentriert ist. Das Recht des „Superfund“ räumt der zuständigen Umweltbehörde einen wciten Ermessensspiclraum ein, um den ausgedehnten Risikopool der kontaminierten Stättc selber zu definieren, aber auch, um innerhalb dieses Pools eine Kerngruppe von Akteuren auszuwählen, dic über die notwendigen Ressourcen und über

48 Weidner, Rehbinder \& Sprenger (1990).

49 Stewart (1991). 
dic notwendige Expertisc für cin effektives Risikomanagement vertügen ${ }^{50}$.

„Bubbles" sind vergleichbare kollektive Risikocinheiten, die durch geographische Grenzen definiert werden. Gruppen von industriellen Luftverschmutzern werden in ciner (un) sichtbaren "Cupola" zusammengefaßt, für die globale Grenzwerte der Belastung festgelegt werden. Innerhalb dieser „bubbles" können dann individuclle Verschmutzungsrechte gehandelt werden ${ }^{5 !}$. Werden aber die Grenzwerte überschritten, treten Probleme kollektiver Haftung auf ${ }^{52}$. Haftung nach Verschmutzungsanteilen - „pollution share liability“ - könnte cine Antwort sein. Interessanter noch sind dic Fäle, in denen der Handel mit Verschmutzungsrechten zu perversen Effekten führt, wenn sich zum Bcispiel "hot spots“ bilden, uncrwünschte lokale Verschmutzungskonzentrationen innerhalb der globalen Grenzen der „bubble“. Die dann entstehenden neuen kollektiven Haftungsprobleme können auf den Staat als den Initiator der „bubble" verlagert werden ${ }^{53}$; mit dem gleichen Recht kann man sie aber auch auf das Verschmutzerkollektiv, auf die „Bubble“ selber verlagern ${ }^{54}$.

In Deutschland läuft cine lebhafte Debatte, ob und wie man regional dezentralisierte Umweltgenossenschaften schaffen könnte, die kollektive Haftung mit kollektivem Risikomanagement verbinden würden ${ }^{55}$. Sie könnten auf dem altbekannten Institut der Wasserverbände aufbauen, die Schäden durch Wassernutzung und Bergbau kollektiv regeln. Sie müßten nur ihren Satzungszweck entsprechend den neuen ökologischen Bedürfnissen neu bestimmen.

"Der Zusammenschluk aller Schadstoffenteiter cines Fluklaufes bew aller Luftverschmutzer cines Rames in je einer Genossenschaft bietet cinzigartige

50 Stewart $(1991), 112$.

51 Dales (1968); Raufer \& Foldman (1987).

52 Keder (1991).

53 Roberts (1982), 1026 ff; Pectors (1991), 162.

54 Boucquey (1904).

55 Bohne (1987); Kinkel (1989), 295 f; Rchbinder (1989), 161; Wagner (1990). 
Möghchkeiten sachaher, regionalen Besonderheiten Rechnung tragender Umwelvorsorye. Da der Eintritt von Umweltschaden in aller Regel eine Frage der Dosis, also der Schatstoffonzentration und des Expositionszeitraums ist und der Grolteil der tmissimen in lmittentennähe niedergeht, wären regiomale Zustandigkeiten im Bereth der Unwelevorsorge empfehlenswert ${ }^{*}$.

Aus dem Zitat wird deutlich, daß regionale und dezentralisierte Risikopools gerade wegen ihrer Eignung für kollektives Risikomanagement bevorzugt werden.

Wenn die Konturen der okologischen „Cupola“ entsprechend dem betroffenen ökologischen Problembereich ausgeformt sind, bleibt die weitere Frage $z u$ klären, welche $A k$ tivitäten von ihr überdeckt werden sollen. Wer ist Mitglied des Risikopools? Wieder scheint mir die Eignung für kollektives Risikomanagement entscheidend zu sein. Es ist kein Zufall, daß die deutsche Rechtsprechung, als sie über die Verantwortlichkeit des Staates für das „Waldsterben“ entschied, im Laufe ihrer Begründung cine kollektive Haftung der Autofahrer nicht in Erwägung $70 \mathrm{~g}$, obwohl Autofahren der wichtigste Ursachenkomplex in diesem ökologischen Problembereich ist ${ }^{57}$. Der Grund für den Ausschluß der wichtigsten "Ursache" ist cine schlichte \%weckmäßigkeitserwägung. Geringfügige Beiträge von Millionen von Autofahrern, die gemeinsam zu cinem schwcrwiegenden Risiko beitragen, schafft eine besondere Risikosituation, die es höchst unzweckmäßig macht, cine kollektive Haftung anzunchmen. Diese Situation ist nicht typisch für die neue kollcktive Haftung, die eine nicht zu große Gruppe von Risikoverursachern zu einem aktiven gemeinsamen Risikomanagement veranlassen könnte, sei es auf der Basis der "deep pocket", sei es auf der Grundlage ciner gemeinsamen institutiondlen Risikokontrolle. Aktives gemeinsames Risikomanagement bezicht sich auf relative kleine "homogen zusammengeset $\%$ te, überschaubare, interaktive Kollektive" 58 . Das führt zu einer Definition der haftungsrechtlichen Risikopools,

56 Wigner $(1990), 112$.

57 BGII/ 102, 350, 362t.

58 Wayner (1990), 109; Kinkel (1989), 296. 
die den Durchschnittsbürger mit kumulierenden Kleinrisiken auschliekit und sich statt dessen auf "corporate, professional, and governmental defendants" konzentriert und so Risiken effektiver vorbeugt und sie besser verteilt ${ }^{59}$.

Ein ähnliches Prinzip findet sich in der market share liablity. Häufig ist die Tendenz zu verspüren, die Haftung nur auf „substantielle" Risikoverursacher zu erstrecken ${ }^{60}$. Das gleiche gilt für die Haftung mehrerer Verschmutzer gemäß $\$ 22$ Wasserhaushaltsgesetz. Der amerikanische „Superfund“ kennt eine „de minimis"-Klausel, die es der zuständigen Behörde erlaubt, sich mit Verursachern, die nur geringfügige Beiträge geleistet haben, finanziell zu vergleichen, und sich auf die großen und mächtigen Mitspieler zu konzentrieren ${ }^{61}$. In all diesen Fällen ist die juristische Haftung an eine bestimmte soziale Handlungsfähigkeit geknüpft.

Schließlich stellt sich das Problem, welche Pflichten den individuellen Mitgliedern der "Cupola" auferlegt werden. Für cinzelne Unternehmen im Pool zeichnen sich heute schon die Umrisse ciner „ökologischen Loyalitätspflicht“ ab, gan\% parallel zu der wohlbekannten gesellschaftsrechtlichen Treuepflicht. Zum Beispiel regelt $\$ 5 \mathrm{BlmSchG}$ eine „ökologische Organisationspflicht ${ }^{\text {"62 }}$. Ahnliche Entwicklungen finden auch oberhalb des Niveaus der Einzelunternehmen statt. Wieder können die „hot spots $^{\text {"i }}$ in „bubbles" als Beispicl dienen ${ }^{63}$. In einem Markt für Verschmutzungsrechte steht es den Teilnehmern frei, so viele Rechte zu kaufen und zu verkaufen wie sie wünschen. Aber der institutionelle Kontext legt ihnen Treuepflichten, „fiduciary duties" auf, die sich aus dem ökologischen Zweck der "bubble“ ableiten lassen. Die „bubble“ ist letzten Endes keine bloße ökonomische Institution, sondern eine ökologische Institution mit dem Ziel, die Umwelt durch cinen Markt für Verschmutzungs-

59 Pricst $(1990), 219$.

60 Sindell v. Abbot Labomatorics, 607 P.2d 924 (C.2. 1980).

$6)$ Stewart (1991), 112.

62 Foldhaus (1991),931.

63 Roberts (1982); Boucquey (1994). 
rechte zu schützen. Dicser Gesetzeszwcck rechtfertigt es, daß die Rechtsprechung Verhaltenspflichten für die Mitglieder der "bubble" entwickelt, dic über die gewöhnlichen deliktisehen Verhaltenspflichten im Markt („lauterer Wettbewerb“) oder die gewöhnlichen Treuepflichten innerhalb ökonomischer Organisationen (,gesellschaftsrechtliche Treupflichten“) hinausgehen. Diese Verhaltenspflichten verbieten es im Falle eines "hot spot" dem einzelnen Mitglied, so viele Verschmutzungsrechte zu kaufen und zu nutzen, daß eine unerträgliche lokale Belastung entsteht, selbst wenn die globalen Grenzwerte der „bubble“ dabei nicht überschritten werden sollten. Ich vermute, daß dic weitere Entwicklung der Risikopools noch zu zahlreichen weiteren Anwendungsfällen ökologischer Treuepflichten für Mitglieder des Pools führen wird.

Das beunruhigendste, gleich zeitig aber auch das vielversprechendste Thema in diescm Zusammenhang heißt: „Solidarität" im Risikopool. ${ }^{64}$. Wir haben gesehen, daß kollektive Haftung bedeutet, einzelne Akteure für Taten verantwortlich zu machen, die andere begangen haben. Wenn wir das in die Sprache der Rechtsflichten übersetzen, dann wird jedem Poolmitylied dic Pflicht auferlegt, das Verhalten der anderen Poolmitglieder zu überwachen. Heißt das nicht, Unmögliches zu verlangen? Selbst solche Akteure, die alles Erdenkliche unternehmen, um das von ihnen selbst gesetzte Risiko zu verringern, können dieser Pflicht nicht entrinnen. Sie haben die unvermeidbare Konsequenz der Lockerung des Kausalitätserfordernisses zu tragen: Ihre individuclle Risikosphäre ist nicht mehr das cinzelne Unternehmen, sondern der ganze Pool. Dies führt natürlich zu Kritik unter Berufung auf Fairneß und Effizienz und verleitet manchen dazu, den ganzen kollcktiven Ansat\% zur ökologischen Haftung zu verdammen (siehe oben). Insbesondere im Rahmen der „Supcrfund"-Haftung erscheint es 
"questionable from an efficiency point of view whether curtent onwers of pices of land who had no influence on pollution should be jomtly and severally liable with the former owners and polluters of the land" ${ }^{\text {wi.5. }}$,

Gleichzeitig scheint dieser beunruhigende Aspekt das Hauptmotiv der Befürworter der Risikohaftung zu sein, die "Cupola“ unsichtbar zu machen.

Es gibt nur cine wirkliche Lösung, nur cine Möglichkeit, dieser Pflicht nachzukommen - durch effektive Zusammenarbeit! Der wesentliche Grund dafür ist im Charakter des "Gutes" zu finden: In unseren Konstellationen sind Verbesserungen der ökologischen Sicherheit

"... a , local public sood" to the industry. A concerted industry effort to improve sofety is requiret since, by definition, the problen is inherent in the nature of the product and is not the result of carelessness by individual producess

Deshalb schafft das kollektive Haftungsrecht letzten Endes de facto - eine Pflicht, zur gemeinsamen Risikokontrolle zusammenzuarbeiten, eine Pflicht, sich zu kollektivem Handeln zu organisieren, cine Pflicht, Institutionen zu schaffen, die das kollektive Management kollektiver Risiken übernehmen, was im Prinzip Präventionsforschung bedeutet.

Im Kontext der Gefährdungshaftung ist es natürlich nicht besonders sinnvoll, von einer "Rechtspflicht" zur Kooperation zu sprechen, da Voraussetzung für die Haftung allein der Schaden ist, ob die Pflicht nun erfüllt wurde oder nicht. Aber selbst in diesem Kontext taucht dic "Pflicht" als cine faktische Oblicgenheit zur Prävention wieder auf. Wer auf cinem Markt tätigr wird, wo cine Haftung nach Marktanteilen gilt, und sein Haftungsrisiko reduziercn möchte, kann sich nicht darauf beschränken, dic Risiken im eigenen Unternehmen zu reduzicren; vielmehr tut er gut daran, sich nach Instanzen kollektiver Uberwachung aller Markttcilnehmer umzuschauen.

Im Zusammenhang der Verschuldenshaftung macht es um so mehr Sinn, den Gedanken einer Kooperationsplicht

65 Ilofstetter (1994), 13.

66 Rose-Ackerman (1990), 745. 
weiterzuverfolgen ${ }^{67}$. Wenn das konkrete Ausmaß der erforderlichen Sorgfalt in einer Situation gemeinsamer Haftung zu bestimmen ist, genügt es nicht, allcin auf die Vorsorge im individuellen Unternchmen abzustellen. Es muß näherbestimmt werden, welche Maknahmen zu ergreifen sind, um dic Risiken in dem gesamten Risikopool zu reduzicren. Das Recht schafft eine "Organisationspflicht", nach der das Management jedes einzelnen Unternehmens sicherzustellen hat, daß sich alle Firmen kollektiv um Risikoreduzierung bemühen, indem sie Personal, Matcrial und technische Abläufe entsprechend organisieren. In dem Zusammenhang sollten freilich Uberlegungen angestellt werden, ob cin adäquater individucller Beitrag zur kollektiven Risikokontrolla genügen kann, um der kollektiven Haftung zu entkommen (s. unter V.).

\section{Auswirkungen auf dic reale Welt}

An dieser Stelle sollten wir allerdings vorsichtiger sein. Denn wenn wir über Vorbeugung und Risikokontrolle sprechen, dann verlassen wir die symbolische Welt des Rechts und beschäfigen uns mit den Auswirkungen des Rechts auf die reale Welt. Diese Bezichung ist durchaus nicht so eng und unmittelbar wie es die Annahme Norm-Sanktion-Gchorsam der traditionellen Rechtswissenschaft voraussetzt. Wir sollten uns auch nicht von der "law-and-cconomics"-Rhetorik verführen lassen, die uns glauben machen will, daß schon kleine Veränderungen im Recht, zum Beispiel eine Änderung des Fahrlässigkeitsmaßstabes, unmittelbar als wirtschaftliche Anreize zur Prävention wirken ${ }^{68}$. Statt dessen sollten wir uns aufmerksam anhören, was empirische Untersuchungen zu den realen Effekten des Rechts und was theorctische Studien zu den komplizierten Beziehungen zwischen Rechtsnormen und wirtschaftlichem Verhalten

67 Siche auch Britzemeier (1994), 4.3.

68 Deutlich bei Thenbers (1989), $308 \mathrm{ft}$ 
zu sagen haben ${ }^{69}$. Sie legen es uns nahe, das über-optimistische Modell "Anreize durch Rechtsnormen" durch das bescheidenere Modell "social order from legal noise" zu ersetzen ${ }^{70}$.

Empirische Untersuchungen über die realen Auswirkungen des Haftungsrechts machen deutlich, dals Wirtschaftsunternehmen Änderungen des Haftungsrechts, selbst so dramatische wic den Ubergang von Verschuldenshaftung zur Gefährdungshaftung, nur als „outside noise", als extrem unbestimmte Botschaften wahrnimmt, und nicht als klare Signale, die zur Feinabstimmung korporativen Verhaltens zwingen, wie es die juristische und ökonomische Literatur gerne annimmt.

"All the firms vicwed product liability as essentially a randon intluence, generating no clear signal as to how to adjust design behavior... we were struck in the companies that we visited by how few changes in law were transmitted to those involved in design decisions" ${ }^{7 !}$.

Soziologische Theorien über die Wechselbezichung von Rechtsnormen, Politikentscheidungen und wirtschaftichem Handeln von Max Weber (1921: 319ff) bis Niklas Luhmann (1988: 324ff) klären uns darüber auf, daß dicse Ubermittlungsschwierigkeiten nicht einfach auf Informationsverluste zurückzulühren sind, die durch Verbesserung der Kommunikation beseitigt werden könnten. Tatsächlich werden wir hier mit kommunikativen Verzerrungen konfrontiert, die aus der inneren Logik der verschiedenen beteiligten Sinn welten resultieren: ökologische Politikprozesse, dic Praxis des Deliktsrechts, dic Dynamik des relevanten Marktes, die interne Politik formaler Organisationen. Kein Zweifel, Recht und Politik senden Signale an die Wirtschaft, die ihre Handlungen an diesen Differenzen auch auszurichten hat.

69 7..B. Weber (1987), McGuire (1988).

70 Zu dieser Formel allgemein IÖrster (1984), Kap. 1; hwogen auf das Recht Teubner (1989), Kap. 5.

71 Eads/Reuter (1983), 107 und IX. 
"Aber dieser Elfekt ist schon nicht mehr Steucrung und auch nicht steuerbar, weil er davon abhängt, was im Kontext anderer Systeme als Differenz. konstruiert wird und unter die dort praktizierten Steuerungsprogramme fällt" ${ }^{\text {72 }}$.

Wirtschaftsunternehmen „verstehen“ Rechtsnormen nicht als gültige normative Gebote, die unbedingten Gehorsam verlangen. Vielmehr nimmt die Welt der Wirtschaft Rechtsnormen extrem selektiv wahr und rekonstruicrt sic in einem vollständig anderen Bedeutungszusammenhang. Entsprechend der internen Logik des konkreten Marktes und des konkreten Unternehmens werden rechtliche Signale neu konstituiert. Im Prinzip rekonstruiert jede dieser Sinnwelten die rechtichen Signale, aber cin und dasselbe rechtliche Signal kann durchaus in einer Vielfalt von ökonomischen Rekonstruktionen wiedererscheinen ${ }^{73}$. Die Auswahl zwischen verschicdenen Rekonstruktionen hängt jeweils von der konkreten Situation ab. In der Welt ökonomischer Transaktionen werden Haftungsregeln auf verschiedene Weisen rekonstruicrt: meist als reine Kostenfaktoren, manchmal als wirtschaftliche Eigentumsrechte, gelegentlich als Verhandlungsmasse - und nur selten als Änderung der Akteurspräterenzen. In der internen Entscheidungswelt von Organisationen wiederum werden sic in anderer Vielfalt verschiedener Bedeutungen rekonstruicrt: als organisatorische Beschränkungen, als interne Machtpositionen, als neue Elemente im "goal sct", als rein rechtliche Probleme, die nur die Juristen angehen, als Kostenfaktoren, die die Finanzabteilung betreffen - und nur selten als Anreize für Manager, dic Uberwachung der Produktion zu ändern oder für Ingcnicure, das Produktdesign zu ändern.

Dies erlaubt, die folgenden Ergebnisse empirischer Untersuchungen, dic optimistische Annahmen über die Erzeugung von Anreizen auf dem Markt widerlegen in einem anderen Lichte zu schen:

"In reality, however, the comnection between the law and product design is sufficiently weak that even quite major changes in the law would have little

72 Luhmann (1988), 337.

73 Daku gemauer Teubner (1991), 531 if. 
effect on the behavior of firms ... exeept to the extent that such change led to significant changes in the overall cost of product claims" 34 .

Innerhalb wirtschaftlicher Organisationen geht die rechtliche Botschaft infolge der typischen Arbeitsteilung zwischen verschiedenen Abteilungen regelmäßig verloren, bevor sie cinen Anreiz bilden kann, andere Entscheidungen zu treffen ${ }^{75}$. In manchen Organisationen fanden empirische Untersuchungen sogar bewulite Strategien, dic „substantial efforts to keep their ... liability problems separate from their ongoing operating decisions" unternahmen ${ }^{76}$.

Nach einer solchen zweifachen kommunikativen „Verzerrung" rechtlicher Botschaften durch den Markt und durch dic Organisation besagen die Signale rechtlicher Haftung bestenfalls: „Be careful or you will be sued“ ${ }^{77}$. Dieses unbestimmte Signal wird sicher nicht regelmäßig in präventive Maßnahmen umgesetzt, sondern in Umgehungsmanöver aller Art entsprechend der jeweils vorherrschenden Strategie der Organisation.

Das Norm-Anreiz-Modell kann diese komplizierten Rekonstruktionsprozesse in verschiedenen Sinnwelten, in ökologischer Politik, im Haftungsrecht, in Markttransaktionen und in der internen Dynamik von Organisationen nicht systematisch erfassen. Es subsumiert sie alle unter die eindimensionale Sprache wirtschaftlicher Kostenüberlegungen, und berücksichtigt Abweichungen in der realen Welt allentalls durch ceterisparibus-Klauseln oder durch Ad-hoc-Anpassungen des Modells an die "Realität“ ${ }^{* 8}$. Daher hat das Norm-Anreiz-Modell nur beschränkten Nutzen für unser Problem, wie sich das Haftungsrecht in der realen Welt auswirkt, wenn es eine neue kollektive ökologische Haftung einführt. Wir ersetzen es besser durch das bescheidenere Modell wiederkehrenden "rechtlichen Drucks" und "korporativer Reaktionen", die ihrerseits wie-

74 Fads/Rcuter (1983), LX.

75 Stonc (1975), $201 \mathrm{ft}$; Scharpt (1987), $117 \mathrm{f}$.

76 Eads/Reuter (1983), 94.

77 Lads/Reuter (1983), VIII.

78 Etwa Tietenberg (1989), $315 \mathrm{ff}$. 
der neuen rechtlichen Druck und neue korporative Reaktionen hervorrufen und so weiter in einem unendlichen selbstorganisierenden Prozess. Wir sollten daher skeptisch im Hinblick auf unsere technischen Fähigkeiten sein, raffinierte Kostenanreize zu ersinnen, dic das Verhalten individueller oder korporativer Akteure in einer Welt ökonomischer Rationalität verändern sollen. Wir rechnen besser mit einer Vielfalt autonomer sclbst-organisierender Prozcsse - etwa der Prozesse ökologischer Politik, des Haftungsrechts, des Produktmarkts und formaler Organisationen - die operativ getrennt ablaufen und doch zur gleichen Zeit strukturell miteinander gekoppelt sind. Sic reagieren aufeinander, aber nur in einer extrem selektiven und eher unvorhersehbaren Weise ${ }^{79}$. „Sustainable development', not efficiency emerges thereby as the ultimate para$\operatorname{digm}^{\text {" }}{ }^{\circ}$.

Ebenso skeptisch sollten wir die prognostischen Fähigkeiten der cleganten soziologischen oder ökonomischen Modelle beurteilen. Konsequenzialismus ist im Haftungsrecht möglich und sinnvoll, aber nicht im Sinn von ex ante-Vorhersagen, als ob das Haftungsrecht mit Hilfe ökonomischer oder soziologischer Modelle effektiv die Effekte seiner Veränderungen vorhersagen könnte und auf diese Effekte durch antizipierte Anpassungen reagieren könnte. Konsequenzialismus ex post erschcint weitaus realistischer in dem Sinne, daß die Institutionen des Haftungsrechts sensibler gegenüber ihren realen Effekten in der Welt der Wirtschaftsorganisationen werden sollten als sie es zur Zcit sind. Sie sollten ihre Konzepte an ihre tatsächlichen Erfahrungen mit korporativen Reaktionen anpassen und neuen rechtlichen Druck und neuc korporative Reaktionen in einem langfristigen "Entdeckungsverfahren" schaffen, das auf dem Prinzip "order from noise" aufbaut.

Was läßt sich dann aber über die Reaktionen der Wirtschaftsunternehmen auf die neue ökologische Risikohaftung noch sagen? Rückzug der Versicherungsindustrie vor den ökologischen

79 Blceher (1994).

80 Hofstetter (1994), 14. 
Risiken? Erfindung neuer Versicherungstechniken, dic an die gesamtschuldnerische Haftung angepaßt sind? Entstehung von Institutionen des kooperativen Risikomanagements? Opportunistisches Verhalten von Unternehmen in Situationen kollektiven Handelns, das zu einem incffizienten Niveau ökologischer Prävention führt? Nichts als defensives Verhalten in Prozeßführungsstrategien? - Empirisches Material existiert durchaus, insbesondere im Bereich der Produkthaftung 81 . Aber insgesamt genügt dieses Material nicht, um schon von stabilen Mustern korporativer Reaktionen sprechen zu können, auf dic das Haftungsrecht seinerseits mit neuen Mafsnahmen rechtichen Drucks reagieren könnte, um dann wiederum neue Reaktionen abzuwarten. In einer solchen unübersichtlichen Situation können wir uns nur auf die Diskussion einzelner Szenarios zurückziehen, in denen wir auf der Basis der volhandenen begrenzten Erfahrung übcr Handlungsmöglichkeiten in parallel ablaufenden juristischen und ökonomischen Prozessen spekulieren können.

\section{Szenario I: Opportunismus - Eigennut' und Tücke}

Wahrend die kollcktive Haftung unter dem Gesichtspunkt ausgleichender Gerechtigkeit schr viel Sinn macht, crzeugt sic doch gleichzeitig Probleme kollektiven Handelns, auf die korporative Akteure möglicherweise opportunistisch reagicren. Wenn Opportunismus die korporative Reaktion gegenüber einem System kollektiver Haftung darstellt, gibt es darauf eine adäquate rechtiche Antwort?

Gegenüber dem strikt individuellen Haftungssystem weist eine Kollektivhaftung folgende Vorteile auf. Erstens dient sie der Ausgleichsfunktion des Haftungsrechts, da die Geschädigten auch in einer Situation entschädigt werden können, in der die Individualhaftung schlicht versagen würde. Z Weitens kann man, da über den Risikopool die Kosten vollständig internali-

81 Eads/Reuter (1983); Weber (1987); McGuire (1988). 
sicrt werden, im Sinne der Steuerungsfunktion des Haftungsrechts eine Reallokation von Ressourcen erwarten. Jedenfalls theoretisch fallen dann alle Kosten auf den Risikopool zurück, was insgesamt die Preise beeinflussen und ökologisch riskante Aktivitäten im gesamten Pool verteuern wird. Drittens kann ein gewisser, wenn auch eher geringer, Abschreckungseffekt erwartet werden. Jedem einzelnen Teilnehmer des Risikopools droht ein bestimmter Anteil an der kollektiven Haftung. Je nachdem besteht dieser Anteil entweder in dem Risiko, auf der ersten Prozeßstufe als Gesamtschuldner in Anspruch genommen zu werden, oder in dem Risiko, in cinem Folgeprozeß auf seinen Anteil verklagt zu werden, oder schließlich in dem Risiko, über die Haftung nach Marktantcilen oder in einer anderen Form direkter Haftungszuweisung ,pro rata in Anspruch genommen zu werden. Diese Situation kann die Risikokalkulation der Akteure becinflussen und kann zu einem höheren Vorsorgeniveau führen. Im Idealfall würde die drohende Haftung in eine gemeinsame Anstrengung der Verschmutzer umgesetzt, die ökologischen Risiken zu minimieren. Aber genau hier stellen sich die berühmten Probleme kollektiven Handelns.

Wenn dic kooperativen Bindungen innerhalb des Pools schwach sind, treten Probleme des "moral hazard“ auf, ähnlich denen einer kollektiven Versicherung, die individuelle Risiken auf cine Risikogemeinschaft verlagert ${ }^{82}$. Wenn sie sich sicher genug fühlen, nicht beobachtet zu werden, werden individuelle Unternehmen ihre spezifischen Risikobeiträge nicht reduzieren, da cine solche Reduktion ihre Haftung nicht im gleichen Maß vermindert. Wcil ihre Haftung vom Verhalten Dritter abhängt, die sie nicht kontrollieren können, würden sie eher weniger für die Vermeidung ökologischer Risiken aufwenden. Im Ergebnis wäre das Niveau individueller Vorsorge niedriger als in einer - allerdings rein hypothetischen - Situation strikt individueller'Zurechnung.

82 Adams(1985), 225 ff; Abraham (1987), 863; Rehbinder (1989), 151; Wagner $(1990), 45 t$. 
Es ist empirisch erhärtet, daß hier eine reale Gefahr liegt. Keeler (1991) zeigte, daß innerhalb einer ,bubble“, cinem Markt für Verschmutzungsrechte, wo ein globaler Grenzwert individuelle Begrenzungen der Verschmutzung ersetzt, das Risiko der Uberschreitung des Standards höher ist als unter einem System individueller Grenzwerte.

Ahnliche Probleme stellen sich für kollektive Vorsorgemaßnahmen. Obwohl es im Interesse cines jeden Poolmitgliedes wäre, sich an der kollektiven Risikovorsorge zu beteiligen, um das Haftungsrisiko und die begleitenden Kosten der Ersatzleistung für alle zu reduzieren, erscheint es unter bestimmten Umständen unwahrscheinlich, daß sic sich so verhalten. Mit Mancur Olson (1965) würde man argumentieren: Wenn die Anzahl der Poolmitglieder groß ist, keine kooperativen Bindungen vorhanden sind, Wettbewerbsbedingungen vorhersehen und "selektive Anreize“ oder massive negative Sanktionen fehlen, worden dic Poolmitglieder keine gemeinsamen Anstrengungen unternchmen, selbst wenn das die Kosten für jedes Poolmitglied senken würde.

Dieses „Olson-Problem" kollektiver Risikokontrolle, zusammen mit dem "moral hazard" individueller Vorsorge hat eine ökonomisch motivierte Kritik verschiedener neuer kollektiver Haftungssysteme geführt - market share, „Superfund CERCLA“ und allgemeiner, der gesamtschuldnerischen $\mathrm{Haf}$ tung bei mehrfacher Kausalität - ausgelöst ${ }^{83}$. Rechtsökonomen hegen cin tiefes Mißtrauen gegenüber kollcktiven Lösungen, selbst dann, wenn sic effektiv der Ausgleichsfunktion dienen und die Ressourcenallokation effizienter machen. Ihre Kritik zielt auf die in der Tat schwache präventive Wirkun Dabei dürfte auch den Kritikern bewußt sein, daß ihre Kritik nur dann trägt, wenn sie kollektive Haftung mit einer streng individualistischen Haftung vergleichen, die gegenüber den hier angesprochenen komplexen ökologischen Fällen aber gerade versagt.

83 Epstein (1985), 1377; Huber (1985), 277; Abrahum (1987), 883 ff; Marino (1991), $672 \mathrm{ff}$. 
Der Präventionsschwäche der kollektiven Haftung kann man zu cinem gewissen Grade abhelfen. An dieser Stelle ist es sinnvoll, über die Re-Individualisierung kollektiver Haftung nachzudenken. Wenn die Wirtschaftsunternehmen auf kollektive ökologische Haftung mit "moral hazard“, ,free-riding" und anderen Formen von Opportunismus, „self-interest seeking with guile“ ${ }^{44}$, reagieren, dann kann das Haftungsrechts scinerseits darauf reagieren, daß es dem individuellen Unternehmen seinen jeweiligen Anteil an der Verschmutzung schmerzhaft vor Augen führt, indem es den internen Verteilungsmaßstab neu bestimmt ${ }^{85}$. Diese reindividualisierenden Bemühungen können natürlich wegen des Zusammenbruchs der individuellen Verursachung nicht das kollektive Element und den begleitenden Risikopool eliminieren, aber sie können auf der Kollcktivierung aufbauend neue Tendenzen zur Individualisierung einführen. Sic können den kollektiven Verlust entsprechend den individucllen Merkmalen der Poolmitglieder neu verteilen.

Die Situation ist der Schaffung cines korporativen Akteurs vergleichbar: Im ersten Schritt (Bildung des Pools) werden Handlungen, Rechte und Verbindlichkeiten dem kollektiven Akteur als solchem zugerechnet. Im zweiten Schritt (Rückgriff) werden Verluste und Gewinne individuell auf dic Mitglieder entsprechend den jeweiligen Beiträgen verteilt. Der Vorteil eines solchen zweistufigen Verfahrens liegt darin, daß es die Vorteile kollektiver Haftung mit den Anreizen eines individualisierenden Rückgriffs verbindet. Die kollektive Haftung stellt sicher, daß dic Opfer Ersatz erhalten, auch wenn keine individuelle Verursachung festgestellt werden kann; die individuelle Verteilung des Rückurriffs crzeugt für die Akteure Anreize, das ökologische Risiko zu verringern.

Es stehen verschiedene Haftungstechniken zur Verfügung, deren Kombination über die Balance zwischen Schadensausgleich, Abschreckung und Allokation entscheidet. Folgende Fragen stellen sich: Ist es sinnvoll, im Schadensersatzprozeß ge-

84 Williamsin $(1985), 47$.

85 Mariac) (1991), 672. 
samtschuldnerische Haftung anzuwenden und den Ausgleich der Schädiger untereinander auf Rückgriffsprozesse zu verlagern? Oder ist es sinnvoller, die individuellen Risikoanteile unmittelbar durch die Gerichte zuzuweisen, wie es bei market share liability geschieht? Sollte man Gefährdungshaftung und Verschuldenshaftung für die Auferlegung kollektiver Haftung und den individuellen Rückgriff kombinieren? Was sind die Kriterien für den individucllen Rückgriff: glciche Anteile pro Kopf, Marktanteile, Risikobeiträge, Verschulden? In der Literatur werden verschiedene Kombinationen dieser Methoden diskutier $^{86}$. Besonders interessant sind die Vorschläge einer "Haftung nach gewichteten Marktanteilen", dic das Kriterium des Marktanteils mit der individuellen Unfallwahrscheinlichkeit kombinieren ${ }^{87}$. Ebenso interessant ist dic Kombination von Verschuldenshaftung und Gefährdungshaftung bei der Haftung nach Marktanteilen: Primär gilt Haftung fahrlässiger Markttcilnchmer, sekundär gilt Gefährdungshaftung entsprechend den Marktanteilen 88 .

Ich würde rechtspolitisch folgende Kombination vorschlagen: Im Verhältnis zwischen dem Geschädigten und dem Risikopool sollte Gefährdungshaftung gelten, da sie die Ausgleichsfunktion fördert. Im Verhältnis zwischen dem Pool und scinen Mitgliedern sollte der Rückgriff soweit wie möglich individualisiert werden, so daß individuelle Anreize zur Risikoverhinderung gestärkt werden. Verschulden sollte hier der entscheidende Maßstab sein; wenn das nicht möglich ist, dann Zuweisung nach individuellen Risikobeiträgen; wenn das nicht möglich ist, Zuweisung nach Verschmutzungsanteilen oder Marktanteilen; wenn auch das nicht möglich ist, gleiche Anteile für alle Mitglieder.

Die Entscheidung zwischen gesamtschuldnerischer Haftung der Poolmitglieder mit anschließendem Rückgriff einerseits und direkter Haftungszuweisung durch die Gerichte im Haftungs-

86 Komhauser/Reves\% (1989), 837 fl, Marino (1991); 134tewis (1985), $531 \mathrm{ff}$.

87 Marino (199l),674.

88 Komhauser/Revesz (1989), $837 \mathrm{ft}$. 
prozel andererscits ist schwierig zu treffen, da beide Methoden schwerwiegende Nachteile aufweisen ${ }^{89}$. Gesamtschuldnerische Haftung ist schr vortcilhaft für die Geschädigten, da sie ihnen die beliebige Auswahl eines Schädigers erlaubt, dem Geschädigten schon vollen Schadensersatz durch nur cin Poolmitglied gewährt, den Haftungsprozeß von der Berechnung der individuellen Anteile im Pool entlastet, und es dem ersten Beklagten überläßt, seinen Rückgriffsanspruch gegenüber den anderen Poolmitgliedern entsprechend ihren Anteilen am Risiko und an Verschulden in einer Serie von Folgeprozessen durchzusetzen. Die Nachteile der gesamtschuldnerischen Haftungliegen in Fairnekproblemen gegenüber dem ersten Beklagten, der mit den vollen Risiken des Haftungsprozesses und der Rückgriffsprozesse belastet wird, und erschreckend hohen Prozeßkosten ${ }^{90}$. Die zweite Methode - Zuweisung der Haftungsanteile durch dic Gerichte unmittelbar im Haftungsprozeß - löst zwar das FairneRproblem und vermeidet die hohen Transaktionskosten der Rückgriffsverfahren. Es belastet aber die Geschädigten mit dem Problem, jedes einzelne Poolmitglied zu identifizieren und $\% u$ verklagen, und mit dem zusätzlichen Problem, den jeweils richtigen Anteil von den einzelnen Poolmitgliedern cinzuklagen, was sich als cine teure Prozedur herausgestellt hat ${ }^{91}$. Im Interesse der ökologisch Geschädigten würde ich die erste Lösung bevorzugen. Wir sollten allerdings im Auge behalten, daß beide Iösungen ein schwerwiegendes FairneBproblem aufweisen und unverhältnismäßig hohe Transaktionskosten verursachen.

All diesen Anstrengungen, den individuellen Anteil so präzise wie möglich zu bestimmen, liegt freilich ein tieferes Problem zugrunde.

"Finc-tuning damage awards to better reflect marginal harms, however, may $b_{x}$ just so much academic hairsplining because of a deep paradox that lurks beneath those cases where dimages are exactly proportional to market share...

89 Rosenbers $(1987), 220 t$.

90 Weber (1989), 14881 .

9 Rose-Ackorman (1990),743 If. 
the efficiency of a market-share test is very limited ... it will not generate efficient caretaking unless firms can collude" ".

Das ist schwer zu schlucken! Einerseits führt dic fortschrittlichste Methode der individucllen Schadenszuweisung zu ineffektiver Prävention; andererseits würden kollusive/kooperative Lösungen, die vielleicht effektiver wären, gegen gehciligte Prinzipien des Wettbewerbsrechts verstoßen. Diese paradoxe Situation ist der Grund, warum Rose-Ackerman (1990: 746) letzten Endes auf das Deliktsrecht verzichtet und sich auf staatliche Regulicrung verläßt, was auch immer deren Nachteile sein mögen ${ }^{93}$.

Aber warum nicht "Kollusion" als dritten Weg zwischen Deliktsrecht und staatlicher Regulation ernstnchmen? Existicrt nicht vielleicht eine Chance, daß Selbstorganisation innerhalb des Risikopools das Fairneßproblem lösen könnte und dic Transaktionskosten, die notwendigerweise anfallen, wenn die Haftungsanteile von einer außenstehenden Autorität - sei es cinem Gericht oder einer Behörde - festgelegt werden, drastisch verringern könnte? Und vor allem, könnte cine solche „Kollusion“ nicht "efficient caretaking bchavior" auslosen ${ }^{94}$ ?

"In effect, the threat of joint and several liability motivates a collaborative solution among tortfiasors. Just as the defendants will collaborate to minimize their joint expenses as if they were a single person or entity in a concert of action case, so a group of independent fims mily apportion liability through conmet to avoid inefficien and unfar effects of joint and scveral liabiliry" 45 .

92 Rose-Ackerman (1990), 745.

93 Sithe auch Mendl (1991).

94 Rose-Ackerman (1990), 745. Freilich stellen sich hier cine Reihe wett bewerbsrechlicher Probleme, die ciner eigenen Untersuchung bedurten.

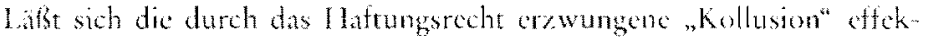
tiv auf ,bkologische" Zusammenmbet beschränken? Whe kann man die Unternehmen daran hindern, in diesem Zusammenhang auch gheichacitig Proisabsprachen ete, zu treffen? Sind es die Vonteile der kollektiven Haftung fur Umweltschaden wert, für ganze Industriczweige das Wettbewerbsrcht einzuschrïnken?

95 Rosenberg (1987), 229 ft. 
Selbstorganisation der Aufteilung des Rückgriffs ist in der Tat eine attraktive Lösung. Wenn der ökologische Risikopool als korporativer Akteur organisiert ist, lassen sich die Nachteile beider besprochenen Lösungen vermeiden. Der Geschädigte braucht nur cinen Schuldner zu verklagen (den Pool oder eines sciner Mitglieder, je nach der juristischen Konstruktion des Pools), kann vollen Ersatz seiner Schäden verlangen und muß sich nicht um die Aufteilung des Schadens unter den Poolmitgliedern kümmern. Die Aufteilung wird durch interne Selbstverwaltung geregelt. Dic Regeln für dic Aufteilung mit Hilfe praktikabler Kriterien lassen sich im voraus vereinbaren. So können beträchtliche Informationskosten und Kosten der Rechtsverfolgung vermieden werden. Die Kosten einer solchen privaten Risikoallokation ,are likely to be less than those entailed by post-accident judicial allocations using either apportioned liability or the increasingly common contribution rule“ ${ }^{\prime \prime}$. In Idealfall werden dic privaten Regeln der Schadenstcilung die Haftung des Pools in cinem solchen Ausmaß reindividualisieren, daß individuelle Anreize für die Prävention geschaffen werden. Aber wie sind die Aussichten für cine kollektive Prävention ökologischer Risiken?

VI. Szenario II - Gemcinsame ökologische Risikoprävention

Ist das nur cin Phantasieprodukt sozio-juristischer Träumer, welche die harten Lehren der Rechtsökonomik nicht begreifen wollen? Es gibt zumindest einige empirische Hinweise, die darauf hindeuten, das manche Reaktionen der realen Wirtschaftsaktcure auf den Druck des Haftungsrechts dem vorherrschenden ökonomischen Zynismus über kooperatives Handeln widersprechen. In einigen Fällen sind Institutionen des gemeinsamen Risikomanagements entstanden - vereimbar oder nicht mit Mancur Olson und scinen autoritären Konsequenzen. Sic haben Aufgaben interner Schadensaufteilung übernommen, und

96 Rosenbery (1987), 231. 
in manchen fällen mehr als das. In manchen Fällen, so wurde berichtet, haben sie die Aktivitäten der Poolmitglieder durch „private Risikoregulierung“ überwacht. Der vielleicht vielversprechendste Aspekt solcher kollektiven Risikoregulierung ist die Aussicht, daß Joint Ventures für ökologische Innovationen entstehen, die integrierte ökologische Technologien an Stelle der herkömmlichen "end-of-the-pipe“-Technologien zu entwickeln in der Lage sind ${ }^{97}$.

Die Chancen für ein durch Haftungsrecht ausgelöstes kollektives Risikomanagement sind jedoch je nach Kontext höchst unterschicdlich. Die Zahl der Akteure, die Struktur des Markts, die Machtbezichungen im Markt, dic Größc des Risikopools, die Intensität der kooperativen Bindungen, die Existenz korporativer Akteure, dic industrielle Kooperation initiieren (Versicherungen, Wirtschaftsverbände), die industrielle kooperative Kultur, dic Rolle öffentlicher Institutionen, dic private Akreure zur Kooperation überreden oder auch zwingen - das scheinen die entscheidenden Faktoren zu sein, welche dic spontane Entwicklung gemeinsamer ökologischer Risikoprävention becinflussen. Es ist eine offene Frage, ob das Recht in der Lage ist, einige dieser Unterschiede zu berücksichtigen, um kooperative Vereinbarungen zu erleichtern und zu unterstützen.

1986 war ein Jahr der ökologischen Desaster - Tschernobyl und Sandoz. 1987 formulierte der Ciba-Geigy-Konzern die korporative Antwort auf eine Welle offentlicher Kritik an der Pharmaindustrie, auf den Verfall ihres öffentlichen Ansehens, auf die Drohung staatlicher Eingriffe und den Druck des Haftungsrechts. Ciba-Geigy startete das sogenannte RAD-AR („Risk Assessment of Druys - Analysis and Response") gemcinsam mit den wichtigsten Pharmaunternehmen. Joint Ventures zum Risikomanagement wurden in jedem der wichtigsten betroffenen Länder gegründet: in den USA, Kanada, Japan, Großbritannien und Deutschland. Eine Arbeitsgruppe war die "Pharmacoepidemiology Group“, deren Aufgabe darin bestand, Informationen über Schäden zu sammeln

97 Siche auch Bruggemeicer (1994). 
und den Zusammenhang zwischen verschriebenen Medikamenten und Schäden. Die andere Arbeitsgruppe war die „Perception/Communication Group". Ihre Aufgabe war die Identifizierung von Risiken und Aufgaben der public relations ${ }^{98}$.

Wir haben hicr die typische Situation cines Oligopols mit nur wenigen mächtigen Akteuren, in der man tatsächlich erwarten kann, daß kollektives Risikomanagement entsteht. Berücksichtigt man die enormen Ressourcen der chemischen Industric, könnte man sich gemeinsame Anstrengungen vorstellen, die über cine reine Versicherungsfunktion hinausgehen, Aufgaben der Erforschung ökologischer Risiken übernehmen und Techniken der Risikoprävention entwickeln, die die Finanzkraft individucller Unternehmen übersteigen.

In dezentralisierten Märkten mit zahlrcichen Akteuren dagegen sind die Chancen eines kollektiven Risikomanagements wesentlich geringer als in einem Oligopol. Kooperation wird nur dann entstchen, wenn bereits vorher kooperative Bindungen existierten". Insbesondere Holdings und lose organisicrte Gruppen unabhängiger Unternehmen mit dezentralisierten Entscheidungsprozessen haben cine Chance, auf den Druck des Haftungsrechts durch die Schaffung eines Risikomanagements jenseits der Unternehmensebene, das sich um ökologische Fragen kümmert, zu reagieren. Die Rechtsökonomik sicht Vorteile in einem solchen Konzern-Risikomanagement gegenuber ciner Kontrolle von außen durch Gerichte oder Behörden: „... monitoring cost for preventing the pollution would basically be lower on the part of the parent than on the part of the political community" 100 . Empirische Untersuchungen legen nahe, daß hier ein fruchtbares Feld für intensive Zusammenarbeit zwischen Unternehmen liegt. Unter dem Druck des Haftungsrechts werden individuelle Unternehmen, die Umweltabteilungen geschaffen haben, dazu neigen, diese Umweltabteilungen von dem einzelnen Unternehmen auf eine höhere

\footnotetext{
98 Burley (1991), 1521

99 Roscuberg (1987), 232.

100 Hotsteter (1994), 13.
} 
Ebene zu verlagern. Im Fall eines Konzerns mit einem hohen Grad interner Arbeitsteilung ist diese Verlagerung besonders wichtig: ,...there is a temptation to belicve that the product as a whole is safe if each subsystem is safe". Kooperative Bemühungen zwischen den einzelnen Unternehmen können daher „help surface cspecially subtlc hazards caused by the interaction of subsystems in a technologically complex product“ ${ }^{101}$.

Wieder anders ist die Situation, wenn der Risikopool entlang vertikaler Linien in ciner Produktionskette oder in ciner ökologischen Kette definiert ist. Hicr ist die Gelegenheit für kooperative Risikokontrolle günstiger, da bereits vertragliche Verbindungen bestehen, die für Vereinbarungen genutzt werden können, die Haftungsrisiken definieren und das Verhalten überwachen. Die Akteure „will in effect comprise an economically interdependent enterprise spanning the cntire chain of production and marketing ${ }^{\omega 102}$. Marketing-Experten sagen eine gesteigerte vertikale Integration als das Ergebnis der neuen $\mathrm{Ri}$ sikohaftung voraus:

"The growth of market share liability could lead to greater cooperation within the channel as well as attempts by the most vulnerable channd members to control channel operations. Thus, the tendency toward vertical marketing systems is likely to be stimulated.

Fewer, harger manufacturer-distributor combinations will be better able to withstand the financial impact of intra-industry joint liability law suits. 'The economies of such large-seale operations may even allow the participaring fims to self-insure should intra-industry risks become unratable. Smaller firms unable to withstand the financial impact of such a suit will be cither forced out of business or compelled to become nembers of substantially larger distribution channels.

Current problens experienced by a channel member in secking indemnifi cation from other menbers will also lad to increased channcl integration....

As a result, manufacturers may begin to monitor the actions of their suppliers more closely, perhaps demanding assurances about the quality of the supplied component or assuming some of the testing and inspecting functions ${ }^{\text {10? }}$.

101 Fads/Reuter (1983), 95.

102 Rosenberg (1987), 230.

103 Boedecker/Morgan (1986),74f. 
Es gibt einige Erfahrungen mit Zulieferernetzwerken und Vertriebsorganisationen, die diese Erkenntnisse bestätigen. Sie beziehen sich auf Situationen, in denen eine vertikale Kette infolge der Existenz eines zentralen Unternchmens, das die gesamte Struktur beherrscht, in cine sternförmige Beziehung umgewandelt wurde ${ }^{104}$. Erfahrungen mit dem „Superfund“ zeigen, daß in einer solchen Situation das zentrale Unternchmen dazu neigt, die Rolle des entscheidenden korporativen Akteurs zu übernehmen, der die gemeinsame Verteilung der Verluste, die Überwachung des Verhaltens der anderen Unternehmen und die Planung der Risikoprärention übernimmt. Wieder spielt das Haftungsrecht eine entscheidende Rolle. Im "Superfund“ sind die finanriellen Risiken so hoch, ist der Risikopool so definiert, daß große Unternehmen fast automatisch diese Rolle übernchmen ${ }^{105}$.

Unter anderen Umständen neigen Wirtschaftsverbände dazu, die Rolle des zentralen Akteurs zu übernehmen. Die in den USA gesammelten Erfahrungen sind nicht gerade überwältigend ${ }^{106}$. Es gibt lediglich einige Reformvorschläge, die Selbstverwaltung ökologiseher Risiken in ganzen Industriebranchen unter staatlicher Aufsicht befürworten, insbesondere die Schaffung branchenweiter Risikofonds ${ }^{107}$. Die europäische Tradition körperschaftlicher Selbstverwaltung kennt allerdings erfolgreiche Beispiele privater Vereinigungen und semi-privater "Quangos" („quasi non-governmental organisations"), die Aufgabe des Risikomanagements und der Risikoprävention übernehmen. Es gibt durchaus ermutigende Beispiele staatlich unterstützter körperschaftlicher Selbstverwaltung auf dem Gebiet der Arbeitssichcrhcit mit cinem sorgfältig ausgearbeitcten Finanzsystem und einer eindrucksvollen Liste „privater Regulierung". Dic deutschen Berufsgenossenschaften sind ein erfolgreiches Beispiel semi-privater Verwaltungskörperschaften, die

104 Rosenberg $(1987), 230$.

105 Stewart (1991), $112 \mathrm{f}$.

106 Rosenbers (1987), 231.

107 Vubank (1991), 21611. 
der heutigen Diskussion über „Umweltgenossenschaften“ als positives Beispicl dienen ${ }^{108}$. Das Problem scheint in der adäquaten Definition des Risikopools zu liegen. Während die Berufsgenossenschaften ihre Risikopools branchenorientiert definieren und zentralisierte Organisationen auf Bundesebene sind, sollen zukünftige Umweltgenossenschaften entsprechend den ökologischen Problemgebieten auf einer dezentralisierten, regionalen Ebene organisiert werden ${ }^{109}$.

Private Versicherungsunternehmen scheinen besonders gut für Aufgaben kollektiven Risikomanagements in cinem ökologischen Problemgebiet gerüstet zu sein ${ }^{110}$. Sic haben professionelle Erfahrung mit Risikoverteilung und können risikorelevante Informationen sammeln, um dic Kosten angemessen auf individueller Basis umzulegen. Es gibt außerdem empirische Beispiele, besonders aus dem Gesundheitssektor, wo Versicherungen tatsächlich cine aktive Rolle bei der Risiküberwachung und der Entwicklung von Techniken der Risikoprävention übernommen haben.

Die neue ökologische Haftung, insbesondere in ihren kollektiven Formen der Unternehmenshaftung, der Haftung nach Marktanteilen und der gesamtschuldnerischen "Superfund"Haftung haben jedoch eine schwere Krise der Versicherungsindustrie ausgelöst ${ }^{111}$. In den USA zog sich die Versicherungsindustrie schlicht von der Versicherung ökologischer Risiken zurück, obwohl die ökologische Versicherung das gewinnträchtigste Geschäft der achtziger Jahre zu werden versprach ${ }^{112}$.

Es ist heute cine offene Frage, ob diese Krise durch eine grundlegende Unvereinbarkeit zwischen kollektiver Haftung, insbesonderc gesamtschuldnerischer Haftung, und Grundprinzipien des Versicherungswesens ausgelöst wurde, wie cinige

\footnotetext{
108 Wagner (1990), 10611.

109 Wagner (1990), $111 \mathrm{ff}$.

110 Abraham (1988), 954 f; Eubank (1991), 174.

111 Eubank (1991), $197 \mathrm{ff}$.

112 Brockett/Golden/Aird (1990).
} 
Autoren annehmen ${ }^{113}$. Es ist ebenso wahrscheinlich, daß die Versicherungsindustrie cine Phase des Experimentierens durchläuft, nach der neue Methoden der Risikoberechnung und vielleicht sogar ein neuer Typ der Versicherungsorganisation entstehen werden. Jedenfalls scheinen im Moment alternative Versicherungsmethoden notwendig zu sein, um die spezifischen Strukturen kollektiver Risiken zu bewältigen. Die „risk retention group" des "Superfund“ ist eine mögliche Antwort, eine weitere wäre eine Pflichtversicherung für die gesamte Branche, eine dritte die Institutionalisierung eines branchenweiten Fonds für ökologische Risiken ${ }^{114}$, cine Lösung, die als besonders geeignet erscheint für atomistische Märkte mit intensivem Wettbewcrb, wo branchenweite Kooperation strukturell unmöglich ist und wo sowohl dic Haftung nach Marktanteilen als auch die gesamtschuldnerische Haftung cher inadäquat erscheinen.

Schließlich scheinen die oben erwähnten Probleme kollektiven Handelns und des "moral hazard“ eine "hybride“ Form der Regulierung notwendig zu machen. In einer Mixtur privatrechtlicher Haftung und offentlich-rechtlicher Regulierung können staatliche Behörden ihre regulatorische Macht mit den Waffen des Haftungsrechts kombinieren, um kollcktive Risikokontrolle zu organisieren. Während derartige hybride Regime von staatlicher Kontrolle privater Selbstregulicrung auf der Unternehmensebene deutliche Fortschritte machen ${ }^{115}$, sind ihre Aussichten auf der Ebene der Unternchmenskooperation noch unklar. Der amerikanische „Superfund“ zur Reinigung kontaminierter Grundstücke ist natürlich das zur Zeit aufregendste Experiment. In unserem Zusammenhang ist dabei ein Punkt entscheidend: Die anfallenden Rechtsverfolgungskosten sind enorm, verglichen mit den Beträgen, die effektiv zur Dekontaminierung verwendet werden ${ }^{116}$. Das unterstreicht die zentrale Bedcutung kollektiver Vereinbarungen - ,vertraglicher Alloka-

113 lubank (1991), $19717,209 \mathrm{ff}$

114 Eubank (1991), $216 \mathrm{f}$.

115 leldhans (1991), $928 \mathrm{ft}$

116 Conment (1988), $28 \%$. 
tion" - die außergerichtlich von den beteiligten Firmen getroffen werden. Wie ein sympathisierender Beobachter bemerkt:

"Ln effect, contract allocation tailors legal regulation of toxic substance risktaking to the individual needs of the partics and context. Its flexibility, in contrast to the more formal and rigid rules of judicial allocation, promises benctits in lower costs, swifter enforcement against cheating, and more protection for confidential information ... legal regulation of toxic substance risks may often be achicved effectively by creating imentives for, and by all means allowing, private contract and enforcenent as an alternative or supplement to centralized command and control decision making by courts and other government agencies " 117 .

\section{Palermo oder Florenz?}

Solche verstreuten Erfahrungen mit "hybriden Regulierungen", die staatlich-politische Steuerung mit privaten kollektiven Vereinbarungen kombinieren, eröfnen eine Perspektive ökologischer neo-korporatistischer Vereinbarungen, die auf statiticher Institutionalisierung und öffentlicher Kontrolle kollektiver Selbstorganisation von Unternehmen beruhen. Die neuen Formen kollekriven Risikomanagements sollen keineswegs individuelle Haftung einerseits oder staatliche Regulierung andererseits vollständig ersetzen. Es geht vielmehr darum, cinen begrenzten und spezifischen Bercich ökologischer Risiken zu definieren, in dem das gemeinsame Risikomanagement privater Akteure individuelle Haftung und statliche Regulierung ergänzen wird. Der Schwerpunkt sind solche Situationen okologischen Risikos, in denen cine individuelle Zurechnung der Verursachung nicht mehr möglich ist und zugleich cine relativ kleine Gruppe von Verschmutzern identifiziert werden kann. In diesen Fällen sollte eine kollektive Haftung der Gruppe mit Anreizen für eine Institutionalisierung kollektiver Risikokontrolle kombiniert werden. Das kollektive Risikomanagement würde Schäden ersetzen, individuelle Risikobeiträge feststellen, riskante Aktivitäten der Gruppenmitglieder überwachen,

117 Rosenberg (1987), 237. 
gemeinsame präventive Maßnahmen crgreifen und technologische Innovationen zur Risikokontrolle in Angriff nehmen. Diese kollektive Haftung kann freilich nicht diffuse ökologische Risiken großen Maßstabes abdecken, die von einer großen Zahl von Akteuren innerhalb großer Zeiträume und/oder ausgedchnter geographischer Räume verursacht werden. In dicsen Fällen sind gesellschaftsumfassende ökologische Fonds und ökologische Steuern deutlich vorzugswürdig ${ }^{118}$. Ebensowenig sollte die klassische individuelle Haftung in Fällen crsetzt werden, wo die kausalen Verbindungen cindeutig identifizierbar sind.

Es sicht so aus, als könnte dic "Cupola" ihre Umrisse verändern. Sic könnte sich von ciner bedrohlichen Hicrarchic der Oko-Mafia in eine die Unwelt schützende Institution entwickeln. Sollte sich dic „Cupola Palermitana“ zur "Cupola Fiorentina" wandeln?

\section{Literatur}

Abraham (1987), Kemneth S.: Individual Action and Collective Responsibility: The Dilemma of Mass Tort Reform, in: Virginia Law Review 73, 845-907. Abraham (1988), Kenneth S.: Environmental Liability and the Linnits of Insurance, in: Columbia Law Kovicw 88, 942-988.

Adams (1985), Michael: Okonomische Analyse der Gefabrdungs-und Verschuldenshaftung, Heidelbern; Deeker \& Schenck.

Adann/Brownsword (1990), John N. und Roger; Privity and the Concept of a Network Contract, in: Legal Studies 10, 12-37.

Assmann (1988), Heinz-Dietcr: Multikausale Schäden in deutschen Haftungsrecht, in: A. Fenyves/H.-L. Weycrs (Hrsg.): Multikausale Schäden in modemen Malingsredten, Lrankturt: Metzner, 99-151.

Bechmann (1990), Gotthard: Grolktechnische Systeme, Risiko und gesellschaftliche Unsicherheit, in: J. Halfmann/K.P. Japp (I Irsg.): Riskante Entscheidungen und Katastrophenpotentiale: Elomente ciner soriologischen Risikoforsebung, Opladen: Westedeutscher Verlay, 123-149.

Bechmann (1991), Gotthard: Risiko als Schlüsselkategoric der Gesellsehaftstheorie, in: Kritiscbe Vierteliahresschrift fir Gesetzgebung und Rechtswisserstebuft 74, 212-240.

II8 Warner (1990); Hohloch (1992). 
Blecher (1994), Michael: Ecological Responsibility of Enterprise: Enviromental Offices in an Fcological-Quality-Organisation, in: ( $\mathrm{s}$. Teubner ( 1 rs Ecological Responsibility, London: Belhaven.

Bodewig (1985), Theo: Probleme alternativer Kausalitat bei Massensehaden, in: Archio fur die coulistische Praxis 185, 505-558.

Boedecker/Morgan (1986), Karl A. und Fred. W.: Intra-industry Joint liabilityImplications for Marketing, in: Journal of Public Policy and Marketing 5, 72 82.

Bohne (1987), Eberhard: Umweltschutzgenossenschaften, Manuskript.

Boucquey (1994), Nathalie: The Other Side of the Bubble: Ecological liability Problems in Markets for Pollution Rights, in: G. Tcubner (Hrsg.): Ecological Responsibility, London: Belhaven.

Brockett/Golden/Aird (1990), Patrick L. und Linda und Paul R.: How Public Policy Can Define the Market Place: The Case of Pollution Liability Insurance in the 1980's, in: Journal of Public Policy and Marketmg 9, 211-226.

Brüggeneier (1991), Gert: Jenseits des Verursacherprinzips? Zur Diskussion un den Kausalitätsnachweis im Unwelthafungsrecht, in: Kritische Vierteljahresschift für Gesetzgebung und Rechtswissenschaft 74, 297-310.

Brüggemeier (1994), Gert: Enterprise I iability for ,Environmental Damage in German and EC-Law, in: G. Tcubner (I Hrsy.): Fological Responsibility, London: Belhaven.

Burlcy (1991), D.M.: Risk Assessment and Responsibility for Injuries Associnted with Medicines, in: G.G. Howells (Hrsg.): Preduct Liability, Instrance and the Pharmaceutical Industry: An Anglo-American Comparison, Manchester: Manchester University Press, 146-155.

Bush (1986), Robert A. Baruch: Between Two Worlds: The Shift from Individual to Group Responsibility in the Law of Causation of hujury, in: Unversity of Califormia of Los Angeles Law Review 33, 1473-1563.

Celli (1990), A.G.: 'Toward a Risk Contribution Approach To 'lortfeasor Identification and Multiple Causation Cases, in: N.Y.U. Law Revice 65, 635-692.

Comment (1988): Liability Insurance Coverage for Superfund Claims: A Mom dest Proposal, in: Moden Law Revtew 53, $2891 \mathrm{f}$.

Dales (1968), John H.: Pollution, Property and Pricts, Toronto: Toronto University Press.

Eads/Reuter (1983), George und Pcter: Designing Safer Products: Corporte Responses to Product Liability Law and Regulation, Santa Monica/California: Rand.

Epstein (1985), Richard: Two Fallacies in Joint Tort Law, in: Georgit Latu Review 73, 1377-1388.

Lubank (1991), Katherine T.: Paying the Cost of I Tazardous Waste Pollution: Why is the Insurance Industry Raising such a Stink, in: University of lllmois Law Review, 173-217.

Foldhaus (1991), Gerhard: Unweltschutssichernde Berrebsergamisation, in: Neue Zeitschrift für Verüaltungsecht, 927-935. 
Förster (1984), Heinz von: Sibt und Einsicht, Braunschweig: Vieweg.

French (1982), Peter A.: Collective Responsibility and the Practice of Medicine, in: Joumal of Medicine and Pbilosophy $7,65 \mathrm{ft}$.

French (1984), Peter A.: Collective and Corporate Responsibility, New York: Columbia University Press.

Hofsteter (1994), Karl: The Ecological Liability of Corporate Groups: Comparing US and European Trends, in: G. Teubncr (Hrsg.): Lological Responsibility, London: Belhaven.

Hohloch (1992), Gerhard: Ausgleich von Umweltschäden in Teilgebicten durch Intschädigungsfonds: Rechtsvergleichende Anmerkungen, in: Umweltrecht $3,73-78$.

Huber (1985), Peter: Safety and the Second Best: The Hazards of Public Risk Management in the Courts, in: Columbiat Law Review 85, 277-337.

Keder (1991), Andrew J.: Noncomplinnt Firms in Transterable Discharge Pcrmit Markets; Some Extensions, in: Jozmal of Enwironmental Lonomies and Managenent 21, 180-189.

King (1981), Joseph H.: Causation, Valuation and Chance in Personal Injury Torts Involving Prexisting Conditions and ruture Consequences, in: Yale law Jounal 90, 1353-1397.

Kinkel (1989), Klaus: Möglichkeiten und Grenzen der Bewäligung von umweltypischen Distanz- und Summationsschäden, in: Zeitschrift fir Rechtspolitik 8, 293-298.

Kornhauser/Reves\% (1989), Lewis A. und Richard L.: Sharing Damages Among Multiple Tortleasors, in: The Yale Law Jommal 98, 831-884.

Köndgen (1991), Johannes: Multiple Causation and Joint Torteasors in Pollution Cases According to German Law, in: J.M. van Dunne (Hrsg.): Trans boundary Pollution and Linbility: The Case of the Ruver Rbine, Rotterdam: Vermande, $99-106$.

L.uhmann (1988), Niklas: Grenzen der Steverung, in: N. Luhmann: Die Wirtsthati der (icscllschalt, liankfurt: Suhrkamp, 324-349.

Luhmann (1991), Niklas: Soziologie des Risikos, Berlin: de Gruyter.

Marino (1991), Anthony M. Market Share Liability and Economic Efficiency, in: Souburn Fonomic Joumal 57,667-675.

MeGuire (1988), Parrick L: The Impact of Product Liability, New York: Conference Broard.

Medicus (1986), Dieter: Zivilrecht und Umwcitschuty, in: Jiristenzeitung 41, $778-786$.

Mendl (1991), Peter S: The Limitations of Logal lnstitutions for Addressing Finvironmental Risks, in: Joumal of Fconomic Perspectives 5, 93-113.

Nickliseh (1991), Fritz: Die Haftung für Risiken des Ungewissen in der jüngsten Gesetzgebunszur Produkt-, Gentechnik-und Umwelthaftung, in: Festschrift fur Hubert Nederländer, Hedelberg: Winter, 341-352.

Olson (1965), Mancur: The Logic of Collectioe Action, Cambridge: Harvard University press. 
Pecters (1991), Marjan G.W.M.: Legal Aspects of Markctable Pollucion P'crmits, in: F. Dietz/ti. van der Ploce/li, van der Stratten (Hrsg.): Lnvirommental Policy and the Lconomy, New York: Elsevier, 151-165.

Podgers (1980), James: DES Ruling Shakes Products Liability Fields, in: American Bar Association Journal 66, $827 \mathrm{ff}$.

Priest (1990), George L.: The New legal Structure of Risk Control, in: Daedalus $119,207-227$.

Prosser/Keeton (1984), William L.. und Page H.: Prosser and Keeton on the Lat of Torts, St. Paul Minn.: West Pub. Co.

Rabin (1987), Robert 1.: Environmental Liability and the 'lort System, in: Houston Law Review 24, 27-52.

Raufer/Feldmann (1987), Roger K. und Stephen 1... Acid Raim and Lmissions Trading: Implementing a Market Approad to Pollution Control, Totowa, N.Y.: Rowman \& littlefield.

Rehbinder (1989), Eckard: Fortentwicklung des Unwelthaftungsrechts in der Bundesrepublik Deutschland, in: Natur und Recht 11, 149-163.

Roberts (1982), Manley W.: A Remedy for the Victims of Pollution Permit Markets, in: The Yale Law Journal 92, 1022-1040.

Robinson (1985), Glen O.: Probabilistic Causation and Compensation for Tortious Risk, in: Joumal of Legal Studies 14, 779-798.

Rose-Ackermann (1990), Susan: Market-Share Allocations in Tort Law: Strength and Weaknesses, The Jourral of legal Studies 19,739-746.

Rosenberg (1984), David: The Causal Connection in Mass lixposure Cases: A ,Public Law Vision of the 'Tort System, in: /latrard Lat Review 97, $851-929$.

Rosenberg (1987), David: Joint and Several liability for Toxic Torts, in: fournal of Hazardous Materials 15, 219-239.

Scharpf (1987), Fritz: Grenzen der institutionellen Reform, in: Jalbrbuch zur Staats-und Verwaltungswissenschaft $1,111-151$.

Schmidt (1991), Eike: Etfizenzbedingungen für privatrechtlichen Sozialschutz, in: Kritische Vierteljahresschrift fur Gesetzgebung und Rechtswissenschaft 74, $378-385$.

Sheincr (1978), Nami: DES and a Proposed Theory of Interprise Liability, in: Fordham Law Revicu 46, 963-1007.

Spitz (1990), Stephen A.: From Res Ipsa lequitur to Diethylstibestrol: The Unidentifiable Tortfeasor in Californis, in: Indiana Law Joumal 65, 591636.

Stewart (199) ), Richard B.: Recent Developments in the Ficld of L iability for Hazardous Waste Under CERCLA and Narumal Reseurce Damage in the United States, in: JM. van Dunné (Hrsg.): Transboundary Pollution and Liability: The Case of the River Rhine, Rotterdam: Vermande, 107-128.

Stone (1975), Christopher: Whore the Law Ends: The Social Control of Corporate Bebavior, New York: Harper \& Row. 
Teubner (1989), Gunther: Recht als autopotetisches System, lrankfurt: Suhrkimp.

Teubner (1991), Gunther: Steuerung durch plurales Recht. Oder: Wie die Politik den normativen Mehrwert der Geldzirkulation abschöpft, in: W. Zapt (Hrsy.): Die Modemisierting der modemen Gesellschaft. Verhandlungen des 35. Deutschen Soriologentages in Prankfurt: Cimpus, 528-551.

Teubner (1994), Gunther (Hrsg.): Ecological Responsibility, London: Belhaven.

Tietenberg (1989), Ton H.: Indivisible Toxic Torts: The Economics of Joint and Several Lability, in: Land Economics 65, 305-319.

Wagner (1990), Gerhard: Kollektives Umwelthaftungsrecht atf genossenschaftlicher Grendlage, Berlin: Duncker \& Humblet.

Weber (1989), Anm D.: Misery Loves Company - Spreading the Costs of the CERCLA Clean-up, in: Vanderbilt Law Review 42, 1469-1509.

Weber (1921), Max: Wirtschaft und Gesellschaft, Tübingen: Mohr (Sicbeck).

Weber (1987), Nathan: Product Liability: The Comporate Response, New York: Conference Board (Report No. 893).

Weidner/Rchbinder/Sprenger (1990), I lelmut und Fekard und Rolf-Ulrich: Dic Unweltpolitik in Japan: Ein Modell für dic EG?, in: IFO-Schnclldienst $N$. 16/17, 33-43.

Williamson (1985), Oliver: The Ecomomic Institutions of Capitalism Fims, Markets, Relational Contracting, New York: Iree Press. 